\title{
Transfer-matrix method based on perturbation expansion for periodic and quasi-periodic binary long-period gratings
}

\author{
G. W. Chern and L. A. Wang \\ Institute of Electro-Optical Engineering, National Taiwan University, Taipei, Taiwan
}

Received January 4, 1999; revised manuscript received June 1, 1999; accepted June 4, 1999

\begin{abstract}
A transfer-matrix method based on perturbation expansion is proposed as an alternative way of simulating the transmission spectrum of a binary long-period grating (LPG). We first generalize the concept of transfer matrices for a heterojunction waveguide. For the couplings among copropagating modes, forward transfer matrices are used to describe the evolution of mode amplitudes along the grating. We show that these elements are related to the well-known coupling coefficients. The method is then used for the study of ideal two-mode grating couplers, and analytic solutions are obtained. We also use the matrix method to study multimode couplings in a LPG and compare the results with those obtained by using the coupled-mode theory. To further demonstrate its usefulness, we apply the method to a special quasi-periodic LPG, the Fibonacci grating. The results show that each cladding mode contributes to several transmission dips and that the dips of different cladding modes are grouped according to the special resonance conditions. (c) 1999 Optical Society of America [S0740-3232(99)00611-0]
\end{abstract}

OCIS codes: $050.2770,060.2340$.

\section{INTRODUCTION}

Since the first demonstration of fiber gratings, ${ }^{1}$ there have been numerous experimental and theoretical papers related to this field. According to the coupling mechanisms, fiber gratings can be classified into two types. One is the short-period grating, conventionally termed the fiber Bragg grating, in which a forward-propagating core mode couples to a backward-propagating mode of the same type. ${ }^{1}$ The other is the long-period grating (LPG), in which a forward-propagating core mode couples to various cladding modes. ${ }^{2}$ The transmission spectrum of a LPG consists of many dips attributed to couplings of the corresponding cladding modes. Many applications utilizing the features of this spectrum have been shown, such as band-rejection filters, ${ }^{2}$ gain equalizers, ${ }^{3}$ and sensors. ${ }^{4}$ The analysis of the transmission spectrum of a LPG is based on the coupled-mode theory. ${ }^{5-7}$ The photoinduced index modulation of the fiber core is assumed to be slowly varying and can be treated as a perturbation. The evolution of the mode amplitudes along the grating is then described by using ordinary differential equations. Numerical integrations such as the Runge-Kutta algorithm are employed to solve the coupled differential equations. If the index modulation is not pure sinusoidal but still periodic, the first-order F ourier component of the modulation is used instead. Note that the coupled-mode theory can also be applied to the analysis of apodized or chirped gratings.

Since the periods of LPG's are large (usually greater than $100 \mu \mathrm{m})$, it is possible to fabricate a binary LPG with an arbitrary pattern. A pure binary LPG is assumed to be composed of two regions. One is the usual fiber structure, which is termed region 0 , and the other is the region exposed to UV irradiation, which is termed re- gion 1. In this paper we assume that the LPG is pure binary and develop a transfer-matrix method to calculate the evolution of mode amplitudes. The core index in region 1 after exposure will be slightly higher than that of the unirradiated core. Thus the modes in region 1 are slightly perturbed from those of region 0 . Using the perturbation expansion and the continuity of electric and magnetic fields, we can connect the mode amplitudes on the two sides of the abrupt interface. Thus, under appropriate assumptions, a forward transfer matrix is used to describe the evolution of the mode amplitudes. The transfer-matrix method has been successfully used in the study of layered structures. ${ }^{8,9}$ The forward- and backward-propagating amplitudes of a mode on the two sides of a heterojunction are connected by using a $2 \times 2$ matrix. A generalized $4 \times 4$ transfer-matrix method has been used by Yeh in the analysis of anisotropic layered media, ${ }^{10}$ in which the matrix is used to connect the forward- and backward-propagating amplitudes of the ordinary and extraordinary waves in each anisotropic layer. For a binary LPG with an arbitrary sequence of regions 0 and 1 , the utilization of a transfer matrix is an efficient tool to study the transmission spectrum of such structures.

This paper is organized as follows. In Section 2 we discuss the formalism of the transfer-matrix method for guided heterostructures. Based on the perturbation expansion, we can derive the transfer matrices from first principles. In a dielectric waveguide, there are continuously distributed modes known as the radiation modes. ${ }^{7,11}$ The existence of such modes makes it impossible to use the matrix method to connect the various mode amplitudes of a heterojunction. Under certain assumptions discussed below, however, the concept of a 
transfer matrix is still applicable for guided heterostructures. We present in Section 3 the forward transfermatrix method and outline the formalism of applying this method to the calculation of the transmission spectrum of a LPG. Section 4 describes the results of two-mode coupling in a periodic binary LPG. The phase-matching condition in our formalism is also discussed. A comparison is made between the coupled-mode theory and our method for the case of ideal two-mode coupling. The effect of duty cycle on the transmission spectrum is also studied. In Section 5 we apply the forward transfermatrix method to multimode coupling in a LPG with the consideration of dispersion. To demonstrate the feature of this method, we use the transfer-matrix method to study the spectrum of quasi-periodic LPG's in Section 6. We will first define the quasi-periodic LPG and discuss the general resonance conditions. Then we will use the transfer-matrix method to study a special quasi-periodic structure, the Fibonacci LPG. The transmission spectrum of the quasi-periodic Fibonacci LPG will be discussed, and each transmission dip will be identified by using the resonance condition. Discussions about the advantages and the limitations of our method will be presented in Section 7. In the final section we make a conclusion about our work.

\section{PERTURBATION EXPANSIONS FOR TRANSFER MATRICES OF OPTICAL WAVEGUIDES}

In this section we will generalize the transfer-matrix method for the application of heterointerfaces of optical waveguides based on the perturbation method. There are various modes in an optical fiber including core modes, cladding modes, and radiation modes. ${ }^{7,11}$ The conventionally referenced fiber modes are the core modes that are guided by the fiber core. If the fiber is surrounded by a medium with a refractive index lower than that of glass, such as air, there are modes guided by the cladding, which are now termed the cladding modes. Besides these discrete modes, there are continuously distributed modes known as the radiation modes, which propagate in directions perpendicular to and along the fiber axis. It can be proved that orthogonality relations hold for these fiber modes ${ }^{11}$ and that an arbitrary field can be expanded by using these sets of modes. The expanding coefficients are known as the amplitudes of the modes. Relations between mode amplitudes of an abrupt interface formed by two waveguides can be deduced by using the boundary conditions of electric and magnetic fields. If the guiding structures of the two waveguides differ slightly, the modes in the two different waveguides are similar to each other. We can thus use the same number to label the modes and use the perturbation method to find the modes of one waveguide in terms of those of the other waveguide. In addition, if we consider mainly the couplings among guided modes, the contribution of radiation modes can be neglected because of the small coupling coefficients of these modes with the guided ones. Under these assumptions the transfer-matrix method can be conceptually generalized as follows.
Consider the heterojunction formed by two slightly different waveguide structures as shown in Fig. 1 . Let the mode fields be $\left(\mathbf{e}_{j}^{(a)}, \mathbf{h}_{j}^{(a)}\right)$ and $\left(\mathbf{e}_{j}^{(b)}, \mathbf{h}_{j}^{(b)}\right)$, respectively, where $\mathrm{j}=1,2, \ldots, \mathrm{N}$. $\mathrm{N}$ is the number of modes. The transverse electric and magnetic fields on each side are

$$
\begin{array}{r}
\mathbf{E}_{\mathrm{t}}^{(\mathrm{r})}=\sum_{\mathrm{j}}\left[\mathrm{A}_{\mathrm{j}}^{(\mathrm{r})} \mathbf{e}_{\mathrm{tj}}^{(\mathrm{r})} \exp \left(\mathrm{i} \beta_{\mathrm{j}} \mathrm{z}\right)+\mathrm{B}_{\mathrm{j}}^{(\mathrm{r})} \mathbf{e}_{\mathrm{tj}}^{(\mathrm{r})} \exp \left(-\mathrm{i} \beta_{\mathrm{j}} \mathrm{z}\right)\right], \\
\mathbf{H}_{\mathrm{t}}^{(\mathrm{r})}=\sum_{\mathrm{j}}\left[\mathrm{A}_{\mathrm{j}}^{(\mathrm{r})} \mathbf{h}_{\mathrm{tj}}^{(\mathrm{r})} \exp \left(\mathrm{i} \beta_{\mathrm{j}} \mathrm{z}\right)-\mathrm{B}_{\mathrm{j}}^{(\mathrm{r})} \mathbf{h}_{\mathrm{tj}}^{(\mathrm{r})} \exp \left(-\mathrm{i} \beta_{\mathrm{j}} \mathrm{z}\right)\right] \\
(\mathrm{r}=\mathrm{a}, \mathrm{b}) .
\end{array}
$$

Using the boundary conditions $\mathbf{E}_{\mathrm{t}}^{(\mathrm{a})}=\mathbf{E}_{\mathrm{t}}^{(\mathrm{b})}$ and $\mathbf{H}_{\mathrm{t}}^{(\mathrm{a})}$ $=\mathbf{H}_{\mathrm{t}}^{(\mathrm{b})}$ at $\mathrm{z}=0$ and the orthogonality conditions

$$
\begin{aligned}
\frac{1}{2} \int_{\mathrm{A}_{\infty}}\left[\mathbf{e}_{\mathrm{i}}^{(\mathrm{r})} \times\right. & \left.\mathbf{h}_{\mathrm{j}}^{(\mathrm{r})^{*}}\right] \cdot \mathbf{z} \mathrm{dA} \\
& =\frac{1}{2} \int_{\mathrm{A}_{\infty}}\left[\mathbf{e}_{\mathrm{i}}^{(\mathrm{r})^{*}} \times \mathbf{h}_{\mathrm{j}}^{(\mathrm{r})}\right] \cdot \mathbf{z} \mathrm{dA}=\mathrm{N}_{\mathrm{j}}^{(\mathrm{r})} \delta_{\mathrm{ij}},
\end{aligned}
$$

we obtain the following relations between the amplitudes of mode fields ${ }^{11,12}$ :

$$
\begin{aligned}
& A_{k}^{(a)}=\sum_{j}\left[\frac{1}{2}\left(I_{k j}+J_{k j}\right) A_{j}^{(b)}+\frac{1}{2}\left(I_{k j}-J_{k j}\right) B_{j}^{(b)}\right], \\
& B_{k}^{(a)}=\sum_{j}\left[\frac{1}{2}\left(I_{k j}-J_{k j}\right) A_{j}^{(b)}+\frac{1}{2}\left(I_{k j}+J_{k j}\right) B_{j}^{(b)}\right],
\end{aligned}
$$

where the coefficients $I_{k j}$ and $J_{k j}$ are defined as

$$
\begin{aligned}
& \mathrm{I}_{\mathrm{kj}}=\frac{1}{2 \mathrm{~N}_{\mathrm{k}}^{(\mathrm{a})}} \int_{\mathrm{A}_{\infty}}\left[\mathbf{e}_{\mathrm{tj}}^{(\mathrm{b})} \times \mathbf{h}_{\mathrm{tk}}^{(\mathrm{a})^{*}}\right] \cdot \mathbf{z} \mathrm{dA}, \\
& \mathrm{J}_{\mathrm{kj}}=\frac{1}{2 \mathrm{~N}_{\mathrm{k}}^{(a)}} \int_{\mathrm{A}_{\infty}}\left[\mathbf{e}_{\mathrm{tk}}^{(\mathrm{a})^{*}} \times \mathbf{h}_{\mathrm{tj}}^{(\mathrm{b})}\right] \cdot \mathbf{z d A} .
\end{aligned}
$$

The generalized transfer matrix can then be symbolically expressed as follows by using Eqs. (3):

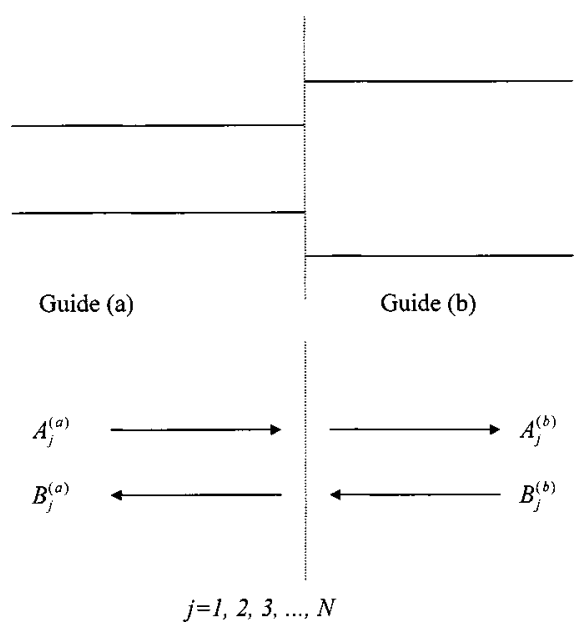

Fig. 1. Schematic diagram of a waveguide heterojunction. The two waveguide structures are slightly different, and the same number is used to label the modes of each waveguide. 


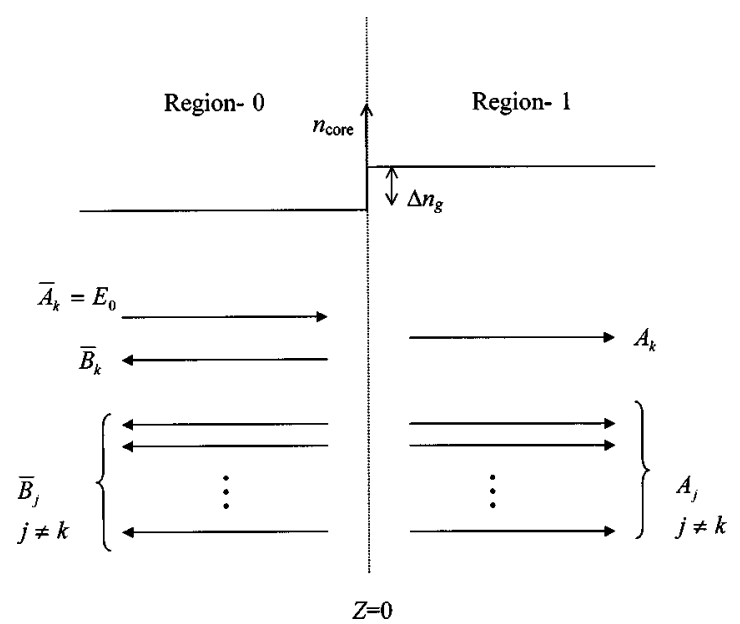

Fig. 2. Incidence of one waveguide mode $k$ on the interface from region 0 to region 1 . Region 1 is formed by UV exposure, and its waveguide structure is assumed to be slightly perturbed from that of region 0 . The incident mode will partially transmit and partially reflect.

$$
\left(\begin{array}{c}
A_{k}^{(a)} \\
B_{k}^{(a)}
\end{array}\right)=\left[\begin{array}{ll}
\frac{1}{2}\left(I_{k j}+J_{k j}\right) & \frac{1}{2}\left(I_{k j}-J_{k j}\right) \\
\frac{1}{2}\left(I_{k j}-J_{k j}\right) & \frac{1}{2}\left(I_{k j}+J_{k j}\right)
\end{array}\right]\left(\begin{array}{c}
A_{j}^{(b)} \\
B_{j}^{(b)}
\end{array}\right) .
$$

We now use the perturbation method to derive the elements of a transfer matrix to connect the mode amplitudes on each side of the 0 and 1 interfaces of a LPG. For clarity, we will henceforward use symbols with a bar above to represent a quantity in the region without UV exposure, which is a 0 in a binary grating, while the unbarred symbol is used for the region with exposure, which is a 1 . We treat the modes of region 1 as being slightly perturbed from the modes of region 0 . The full vectorial perturbation expansion of waveguide modes is derived in Appendix A based on the reciprocity theorem. ${ }^{11}$ The parameter $\mu$ will be used to keep trace of the perturbation expansion. Now consider the situation schematically shown in Fig. 2. Here only mode $k$ of region 0 is incident on the interface with amplitude $\mathrm{E}_{0}$. Let the mode fields in region 0 be the unperturbed ones (cf. Appendix $A$ )

$$
\overline{\mathbf{e}}_{\mathrm{j}}=\mathbf{e}_{\mathrm{j}}^{(0)}, \quad \overline{\mathbf{h}}_{\mathrm{j}}=\mathbf{h}_{\mathrm{j}}^{(0)},
$$

and let the mode fields in region 1 be written as

$$
\mathbf{e}_{\mathrm{j}}=\mathbf{e}_{\mathrm{j}}^{(0)}+\mu \delta \mathbf{e}_{\mathrm{j}}, \quad \mathbf{h}_{\mathrm{j}}=\mathbf{h}_{\mathrm{j}}^{(0)}+\mu \delta \mathbf{h}_{\mathrm{j}} .
$$

Then using the boundary conditions ( $z=0$ is chosen as the interface), we obtain the following equations:

$$
\begin{aligned}
\left(\mathrm{E}_{0}+\overline{\mathrm{B}}_{\mathrm{k}}\right) \overline{\mathbf{e}}_{\mathrm{tk}}+\sum_{\mathrm{j} \neq \mathrm{k}} \overline{\mathrm{B}}_{\mathrm{j}} \overline{\mathbf{e}}_{\mathrm{tj}}= & \mathrm{A}_{\mathrm{k}}\left(\overline{\mathbf{e}}_{\mathrm{tk}}+\mu \delta \mathbf{e}_{\mathrm{tk}}\right) \\
& +\sum_{\mathrm{j} \neq \mathrm{k}} \mathrm{A}_{\mathrm{j}}\left(\overline{\mathbf{e}}_{\mathrm{tj}}+\mu \delta \mathbf{e}_{\mathrm{tj}}\right), \\
\left(\mathrm{E}_{0}-\overline{\mathrm{B}}_{\mathrm{k}}\right) \overline{\mathbf{h}}_{\mathrm{tk}}-\sum_{\mathrm{j} \neq \mathrm{k}} \overline{\mathrm{B}}_{\mathrm{j}} \overline{\mathbf{h}}_{\mathrm{tj}}= & \mathrm{A}_{\mathrm{k}}\left(\overline{\mathbf{h}}_{\mathrm{tk}}+\mu \delta \mathbf{h}_{\mathrm{tk}}\right) \\
& +\sum_{\mathrm{j} \neq \mathrm{k}} \mathrm{A}_{\mathrm{j}}\left(\overline{\mathbf{h}}_{\mathrm{tj}}+\mu \delta \mathbf{h}_{\mathrm{tj}}\right) .
\end{aligned}
$$

By use of the orthogonality relations for $\left(\overline{\mathbf{e}}_{\mathrm{ij}}, \overline{\mathbf{h}}_{\mathrm{ij}}\right)$, the following relations are derived:

$$
\begin{aligned}
& \mathrm{E}_{0}+\overline{\mathrm{B}}_{\mathrm{k}}=\left(1+\mu \alpha_{\mathrm{kk}}\right) \mathrm{A}_{\mathrm{k}}+\mu \sum_{\mathrm{j} \neq \mathrm{k}} \alpha_{\mathrm{jk}} \mathrm{A}_{\mathrm{j}}, \\
& \mathrm{E}_{0}-\overline{\mathrm{B}}_{\mathrm{k}}=\left(1+\mu \beta_{\mathrm{kk}}\right) \mathrm{A}_{\mathrm{k}}+\mu \sum_{\mathrm{j} \neq \mathrm{k}} \beta_{\mathrm{jk}} \mathrm{A}_{\mathrm{j}} \\
& \overline{\mathrm{B}}_{\mathrm{j}}=\left(1+\mu \alpha_{\mathrm{jj}}\right) \mathrm{A}_{\mathrm{j}}+\mu \alpha_{\mathrm{kj}} \mathrm{A}_{\mathrm{k}}+\mu \sum_{\mathrm{i} \neq \mathrm{j}, \mathrm{k}} \alpha_{\mathrm{ij}} \mathrm{A}_{\mathrm{i}} \\
& \quad(\mathrm{j} \neq \mathrm{k}), \\
& -\overline{\mathrm{B}}_{\mathrm{j}}=\left(1+\mu \beta_{\mathrm{jj}}\right) \mathrm{A}_{\mathrm{j}}+\mu \beta_{\mathrm{kj}} \mathrm{A}_{\mathrm{k}}+\mu \sum_{\mathrm{i} \neq \mathrm{j}, \mathrm{k}} \mathrm{B}_{\mathrm{ij}} \mathrm{A}_{\mathrm{i}} \\
& \quad(\mathrm{j} \neq \mathrm{k}),
\end{aligned}
$$

where the coefficients $\alpha_{\mathrm{jk}}$ and $\beta_{\mathrm{jk}}$ are defined as

$$
\begin{aligned}
& \alpha_{\mathrm{jk}}=\frac{1}{2 \overline{\mathrm{N}}_{\mathrm{k}}} \int_{\mathrm{A}_{\infty}}\left(\delta \mathbf{e}_{\mathrm{tj}} \times \overline{\mathbf{h}}_{\mathrm{tk}}^{*}\right) \cdot \mathbf{z} \mathrm{dA}, \\
& \beta_{\mathrm{jk}}=\frac{1}{2 \overline{\mathrm{N}}_{\mathrm{k}}} \int_{\mathrm{A}_{\infty}}\left(\overline{\mathbf{e}}_{\mathrm{tk}}^{*} \times \delta \mathbf{h}_{\mathrm{tj}}\right) \cdot \mathbf{z} \mathrm{dA} .
\end{aligned}
$$

All the complex amplitudes of the mode fields on each side are also perturbatively expanded, by using the $\mu$ parameter, as

$$
\begin{aligned}
& \overline{\mathrm{B}}_{j}=\overline{\mathrm{B}}_{\mathrm{j}}^{(0)}+\mu \overline{\mathrm{B}}_{\mathrm{j}}^{(1)}+\mu^{2} \overline{\mathrm{B}}_{\mathrm{j}}^{(2)}+\cdots, \\
& \mathrm{A}_{\mathrm{j}}=\mathrm{A}_{\mathrm{j}}^{(0)}+\mu \mathrm{A}_{\mathrm{j}}^{(1)}+\mu^{2} \mathrm{~A}_{\mathrm{j}}^{(2)}+\cdots .
\end{aligned}
$$

The zero-order amplitudes are obtained by substituting Eqs. (11) into Eqs. (9):

$\mu^{0}$ order:

$$
\begin{aligned}
& A_{k}^{(0)}=E_{0}, \quad \bar{B}_{k}^{(0)}=0, \\
& A_{j}^{(0)}=\bar{B}_{j}^{(0)}=0 .
\end{aligned}
$$

This is a trivial situation. There is no heterointerface and thus no coupling or reflection at all. Similarly, the first-order amplitudes are obtained by comparing the coefficients of $\mu^{1}$ : 
$\mu^{1}$ order:

$$
\begin{aligned}
& \mathrm{A}_{\mathrm{k}}^{(1)}=-\frac{1}{2}\left(\alpha_{\mathrm{kk}}+\beta_{\mathrm{kk}}\right) \mathrm{E}_{0}, \\
& \overline{\mathrm{B}}_{\mathrm{k}}^{(1)}=\frac{1}{2}\left(\alpha_{\mathrm{kk}}-\beta_{\mathrm{kk}}\right) \mathrm{E}_{0}, \\
& \mathrm{~A}_{\mathrm{j}}^{(1)}=-\frac{1}{2}\left(\alpha_{\mathrm{kj}}+\beta_{\mathrm{kj}}\right) \mathrm{E}_{0} \quad(\mathrm{j} \neq \mathrm{k}), \\
& \overline{\mathrm{B}}_{\mathrm{j}}^{(1)}=\frac{1}{2}\left(\alpha_{\mathrm{kj}}-\beta_{\mathrm{kj}}\right) \mathrm{E}_{0} \quad(\mathrm{j} \neq \mathrm{k}) .
\end{aligned}
$$

The coefficients $\alpha_{\mathrm{kj}}$ and $\beta_{\mathrm{kj}}$ can be evaluated if we use a first-order perturbation correction for $\delta \mathbf{e}_{\mathrm{j}}$ and $\delta \mathbf{h}_{\mathrm{j}}$ (cf. Appendix A). By Eqs. (10), (A2), and (A3), we have

$$
\alpha_{\mathrm{kj}}=\mathrm{a}_{\mathrm{kj}}+\mathrm{b}_{\mathrm{kj}}-\delta_{\mathrm{kj}}, \quad \beta_{\mathrm{kj}}=\mathrm{a}_{\mathrm{kj}}-\mathrm{b}_{\mathrm{kj}}-\delta_{\mathrm{kj}},
$$

where $a_{k j}$ and $b_{k j}$ are defined in Appendix $A$ and $\delta_{\mathrm{kj}}$ is the Kronecker delta symbol. Thus, to first order, we have the following solution for incidence of mode $\mathrm{k}$ from region 0 to region 1 :

$$
\begin{aligned}
& \mathrm{A}_{\mathrm{k}}=\left(1-\frac{\mu^{2}}{2} \mathrm{D}_{\mathrm{k}}\right) \mathrm{E}_{0}, \\
& \overline{\mathrm{B}}_{\mathrm{k}}=\mu \mathrm{b}_{\mathrm{kk}}^{(1)} \mathrm{E}_{0}, \\
& \mathrm{~A}_{\mathrm{j}}=-\mu \mathrm{a}_{\mathrm{kj}}^{(1)} \mathrm{E}_{0} \quad(\mathrm{j} \neq \mathrm{k}), \\
& \overline{\mathrm{B}}_{\mathrm{j}}=\mu \mathrm{b}_{\mathrm{kj}}^{(1)} \mathrm{E}_{0} \quad(\mathrm{j} \neq \mathrm{k}),
\end{aligned}
$$

where expressions for $D_{k}, b_{k k}^{(1)}, a_{k j}^{(1)}$, and $b_{k j}^{(1)}$ are also given in Appendix A. The physical meaning of these coefficients can be interpreted as follows. The incidence of mode $k$ on the heterointerface will partially transmit and partially reflect. The transmission and reflection coefficients are $1-\mu^{2} D_{k} / 2$ and $\mu b_{k k}^{(1)}$. Additionally, the incident field will also excite other forward- and backwardpropagating modes within the two waveguides with the corresponding coupling coefficients $-\mu \mathrm{a}_{\mathrm{kj}}^{(1)}$ and $\mu \mathrm{b}_{\mathrm{kj}}^{(1)}$, respectively, for mode $j$ other than $k$. It can be proved by using formulas (A7)-(A17) below that the total power is conserved to second order in $\mu$ :

$$
E_{0}^{2}-\left|\bar{B}_{k}\right|^{2}-\sum_{j \neq k}\left|\bar{B}_{j}\right|^{2}=\left|A_{k}\right|^{2}+\sum_{j \neq k}\left|A_{j}\right|^{2} \text {. }
$$

Now we consider another case as schematically shown in Fig. 3. The only nonvanishing incident mode is mode $k$ of region 1, i.e., $A_{k}=E_{1}$. Using the same method as that described for the case shown in Fig. 2, we have the following solution for incidence of mode $k$ from region 1 to region 0:

$$
\begin{aligned}
& \bar{A}_{k}=\left(1-\frac{\mu^{2}}{2} D_{k}\right) E_{1}, \\
& B_{k}=-\mu b_{k k}^{(1)} E_{1}, \\
& \bar{A}_{j}=\mu a_{k j}^{(1)} E_{1} \quad(j \neq k), \\
& B_{j}=-\mu b_{k j}^{(1)} E_{1} \quad(j \neq k) .
\end{aligned}
$$

The first-order field expansion coefficients are related to the well-known coupling coefficients. ${ }^{5-7,11}$ The coupling coefficients can, in general, be split into two parts. One is the coupling among transverse mode fields, and the other is that among the z-component mode fields:

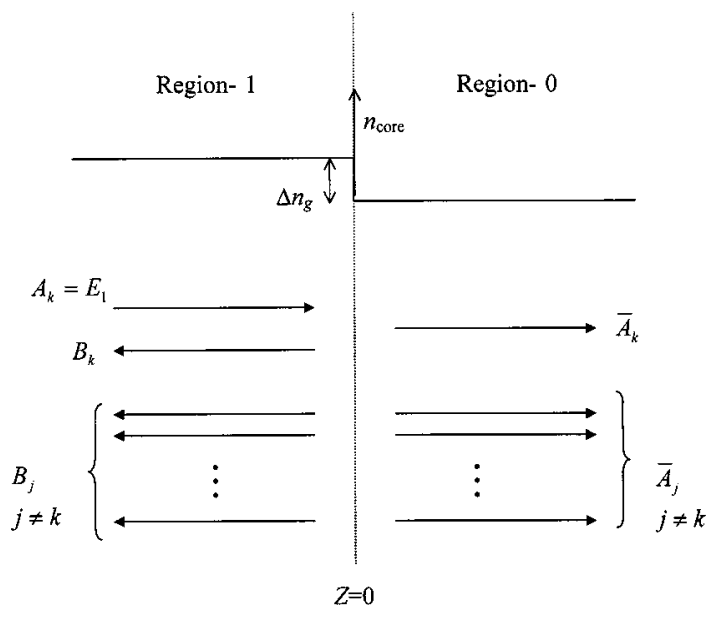

Fig. 3. Incidence of mode $\mathrm{k}$ from region 1 to region 0 , opposite to that shown in Fig. 2.

$$
\kappa_{\mathrm{kj}}=\kappa_{\mathrm{kj}}^{\mathrm{t}}+\kappa_{\mathrm{kj}}^{\mathrm{z}}, \quad \kappa_{\mathrm{kj}}^{\prime}=\kappa_{\mathrm{kj}}^{\mathrm{t}}-\kappa_{\mathrm{kj}}^{\mathrm{z}},
$$

where the transverse and z-component coupling coefficients are defined as

$$
\begin{aligned}
& \kappa_{\mathrm{kj}}^{\mathrm{t}}=\frac{\mathrm{k}_{0}}{4}\left(\frac{\epsilon_{0}}{\mu_{0}}\right)^{1 / 2} \int_{\mathrm{A}_{\infty}}\left(\mathrm{n}^{2}-\overline{\mathrm{n}}^{2}\right) \mathbf{e}_{\mathrm{tk}}^{(0) *} \cdot \mathbf{e}_{\mathrm{tj}}^{(0)} \mathrm{dA}, \\
& \kappa_{\mathrm{kj}}^{\mathrm{z}}=\frac{\mathrm{k}_{0}}{4}\left(\frac{\epsilon_{0}}{\mu_{0}}\right)^{1 / 2} \frac{\mathrm{n}^{2}}{\mathrm{n}^{2}} \int_{\mathrm{A}_{\infty}}\left(\mathrm{n}^{2}-\overline{\mathrm{n}}^{2}\right) \mathrm{e}_{\mathrm{zk}}^{(0)^{*}} \mathrm{e}_{\mathrm{zj}}^{(0)} \mathrm{dA} .
\end{aligned}
$$

By comparing with Eqs. (A7) and (A9), we have the following relations:

$$
\begin{aligned}
& \mathrm{a}_{\mathrm{jk}}^{(1)}=\frac{1}{\beta_{\mathrm{j}}^{(0)}-\beta_{\mathrm{k}}^{(0)}}\left(\kappa_{\mathrm{kj}}^{\mathrm{t}}+\kappa_{\mathrm{kj}}^{\mathrm{z}}\right)=\frac{\kappa_{\mathrm{kj}}}{\beta_{\mathrm{j}}^{(0)}-\beta_{\mathrm{k}}^{(0)}}, \\
& \mathrm{b}_{\mathrm{jk}}^{(1)}=\frac{-1}{\beta_{\mathrm{j}}^{(0)}+\beta_{\mathrm{k}}^{(0)}}\left(\kappa_{\mathrm{kj}}^{\mathrm{t}}-\kappa_{\mathrm{kj}}^{\mathrm{z}}\right)=\frac{-\kappa_{\mathrm{kj}}^{\prime}}{\beta_{\mathrm{j}}^{(0)}+\beta_{\mathrm{k}}^{(0)}} .
\end{aligned}
$$

We now consider the coupling between two specific modes, e.g., modes $\mathrm{j}$ and $\mathrm{k}$. Assume that the coupling to the other modes is insignificant because of unmatched phase, weak-coupling coefficients, etc. The generalized $4 \times 4$ transfer matrix then has the following form:

$$
\left(\begin{array}{c}
\bar{A}_{k} \\
\bar{B}_{k} \\
\bar{A}_{j} \\
\bar{B}_{j}
\end{array}\right)=\left[\begin{array}{llll}
F_{11} & F_{12} & F_{13} & F_{14} \\
F_{21} & F_{22} & F_{23} & F_{24} \\
F_{31} & F_{32} & F_{33} & F_{34} \\
F_{41} & F_{42} & F_{43} & F_{44}
\end{array}\right]\left(\begin{array}{l}
A_{k} \\
B_{k} \\
A_{j} \\
B_{j}
\end{array}\right) .
$$

The elements of the transfer matrix are derived by using the results of Eqs. (15) and (17). The detail of the derivation is left to Appendix B, and the explicit expressions for matrix elements up to second order in $\mu$ are also listed in Appendix B. Additionally, the free propagation matrix over a region of length $L$ is 


$$
\begin{aligned}
& \left(\begin{array}{c}
\mathrm{A}_{k}(\mathrm{~L}) \\
\mathrm{B}_{\mathrm{k}}(\mathrm{L}) \\
\mathrm{A}_{\mathrm{j}}(\mathrm{L}) \\
\mathrm{B}_{\mathrm{j}}(\mathrm{L})
\end{array}\right) \\
& =\left[\begin{array}{cccc}
\exp \left(\mathrm{i} \beta_{\mathrm{k}} \mathrm{L}\right) & 0 & 0 & 0 \\
0 & \exp \left(-\mathrm{i} \beta_{\mathrm{k}} \mathrm{L}\right) & 0 & 0 \\
0 & 0 & \exp \left(\mathrm{i} \beta_{\mathrm{j}} \mathrm{L}\right) & 0 \\
0 & 0 & 0 & \exp \left(-\mathrm{i} \beta_{\mathrm{j}} \mathrm{L}\right)
\end{array}\right] \\
& \quad \times\left(\begin{array}{c}
\mathrm{A}_{\mathrm{k}}(0) \\
\mathrm{B}_{\mathrm{k}}(0) \\
\mathrm{A}_{\mathrm{j}}(0) \\
\mathrm{B}_{\mathrm{j}}(0)
\end{array}\right),
\end{aligned}
$$

where $\beta_{\mathrm{k}}$ and $\beta_{\mathrm{j}}$ are the corresponding propagation constants in this region. By cascading these transfer matrices and using appropriate boundary conditions, we can deduce the transmission and reflection coefficients of a layered structure.

\section{FORWARD TRANSFER MATRICES AND MULTIMODE COUPLINGS}

The $4 \times 4$ transfer matrix (22) takes into account the coupling of both forward- and backward-propagating modes. From the first-order results (15) and (17), we can see that propagation constants are added in the denominator of the reflection coefficients, $b_{\mathrm{j} j}^{(1)}, b_{\mathrm{kk}}^{(1)}, b_{\mathrm{jk}}^{(1)}$, and $b_{\mathrm{kj}}^{(1)}$, while they are subtracted in the denominator of the transfer matrix for the two coupling modes: for incidence from region 0 to region 1 :

$$
\left(\begin{array}{c}
\mathrm{A}_{\mathrm{k}} \\
\mathrm{A}_{\mathrm{j}}
\end{array}\right)=\left[\begin{array}{cc}
\delta_{\mathrm{k}} & \gamma \\
-\gamma^{*} & \delta_{\mathrm{j}}
\end{array}\right]\left(\begin{array}{c}
\overline{\mathrm{A}}_{\mathrm{k}} \\
\overline{\mathrm{A}}_{\mathrm{j}}
\end{array}\right) \equiv \mathbf{F}^{(1 \mid 0)}\left(\begin{array}{c}
\overline{\mathrm{A}}_{\mathrm{k}} \\
\overline{\mathrm{A}}_{\mathrm{j}}
\end{array}\right) ;
$$

for incidence from region 1 to region 0 :

$$
\left(\begin{array}{c}
\bar{A}_{k} \\
\bar{A}_{j}
\end{array}\right)=\left[\begin{array}{cc}
\delta_{k} & -\gamma \\
\gamma^{*} & \delta_{j}
\end{array}\right]\left(\begin{array}{c}
A_{k} \\
A_{j}
\end{array}\right) \equiv \mathbf{F}^{(0 \mid 1)}\left(\begin{array}{c}
A_{k} \\
A_{j}
\end{array}\right) .
$$

Here, for convenience, we have defined the following variables [note that $a_{j \mathrm{k}}^{(1)}=-a_{\mathrm{kj}}^{(1)}$ ]:

$$
\begin{aligned}
& \delta_{\mathrm{k}} \equiv 1-\mu^{2} \mathrm{D}_{\mathrm{k}} / 2, \\
& \delta_{\mathrm{j}} \equiv 1-\mu^{2} \mathrm{D}_{\mathrm{j}} / 2, \\
& \gamma \equiv \mu \frac{\kappa_{\mathrm{kj}}}{\beta_{\mathrm{k}}^{(0)}-\beta_{\mathrm{j}}^{(0)}}=\mu \mathrm{a}_{\mathrm{jk}}^{(1)} .
\end{aligned}
$$

These two coupling processes are schematically shown in Fig. 4. The coupling between two waveguide modes occurs in each of the interfaces of the 0 and 1 regions in a binary LPG. These interfaces act as distributed couplers, with amplitude splitting ratio $\eta_{\mathrm{jk}}$ representing the coupling from mode $j$ to mode $k$. The appropriate expressions for $\eta_{\mathrm{jk}}$ 's for the two interfaces are listed in the figure.

As for the free propagation of these two modes through the waveguide in region 0 and region 1 , we also define two free propagation transfer matrices as

$$
\begin{aligned}
\mathbf{P}^{(0)} & =\left[\begin{array}{cc}
\exp \left[\mathrm{i} \beta_{\mathrm{k}}^{(0)} \Lambda^{(0)}\right] & 0 \\
0 & \exp \left[\mathrm{i} \beta_{\mathrm{j}}^{(0)} \Lambda^{(0)}\right]
\end{array}\right] \equiv\left[\begin{array}{cc}
\exp \left[\mathrm{i} \theta_{\mathrm{k}}^{(0)}\right] & 0 \\
0 & \exp \left[\mathrm{i} \theta_{\mathrm{j}}^{(0)}\right]
\end{array}\right], \\
\mathbf{P}^{(1)} & =\left[\begin{array}{cc}
\exp \left\{\mathrm{i}\left[\beta_{\mathrm{k}}^{(0)}+\mu \beta_{\mathrm{k}}^{(1)}\right] \Lambda^{(1)}\right\} & \exp \left\{\mathrm{i}\left[\beta_{\mathrm{j}}^{(0)}+\mu \beta_{\mathrm{j}}^{(1)}\right] \Lambda^{(1)}\right\}
\end{array}\right] \equiv\left[\begin{array}{cc}
\exp \left[\mathrm{i} \theta_{\mathrm{k}}^{(1)}\right] & 0 \\
0 & \exp \left[\mathrm{i} \theta_{\mathrm{j}}^{(1)}\right]
\end{array}\right] .
\end{aligned}
$$

transmission coefficients, $a_{j j}^{(1)}, a_{k k}^{(1)}, a_{j k}^{(1)}$, and $a_{k j}^{(1)}$. Under the perturbation assumption, therefore, the reflection coefficients are relatively small compared with the transmission coefficients. Thus, to first-order approximation, we can consider only the codirectional coupling. From Eqs. (15) and (17), we obtain the following $2 \times 2$ forward

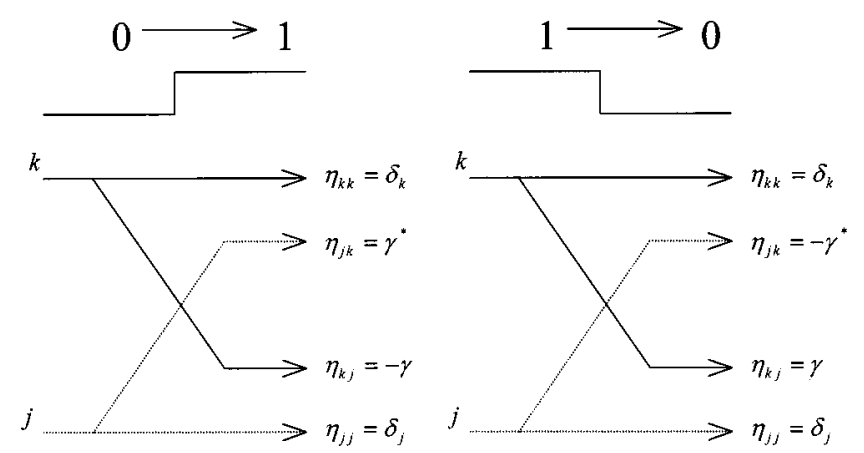

Fig. 4. Schematic diagram showing the distributed coupling between two waveguide modes that occurs in each interface of the 0 and 1 regions in a binary LPG. The interface acts as a splitter with the amplitude splitting ratios shown in the diagram.
Here we use superscripts (0) and (1) on the transfer matrix $\mathbf{P}$ and the phase shift $\theta$ to represent the corresponding quantities in region 0 and region $1 . \Lambda^{(0)}$ and $\Lambda^{(1)}$ are the lengths of region 0 and region 1 , respectively. As depicted in Fig. 5, for a periodic binary LPG, the transfer matrix of a unit period is

$$
\mathbf{F}=\mathbf{P}^{(0)} \mathbf{F}^{(0 \mid 1)} \mathbf{P}^{(1)} \mathbf{F}^{(1 \mid 0)}
$$

Therefore the mode amplitudes of adjacent unit cells are connected through the forward transfer matrix:

$$
\left(\begin{array}{c}
A_{k}(n+1) \\
A_{j}(n+1)
\end{array}\right)=\mathbf{F}\left(\begin{array}{l}
A_{k}(n) \\
A_{j}(n)
\end{array}\right),
$$

where $A_{k}(n)$ and $A_{j}(n)$ are the complex field amplitudes after $n$ unit cells are propagated and $A_{k}(0)$ and $A_{j}(0)$ are the initial field amplitudes. Substituting Eqs. (24)-(28) into Eq. (29), we have the following expression for the unit transfer matrix: 
0 1 0 1
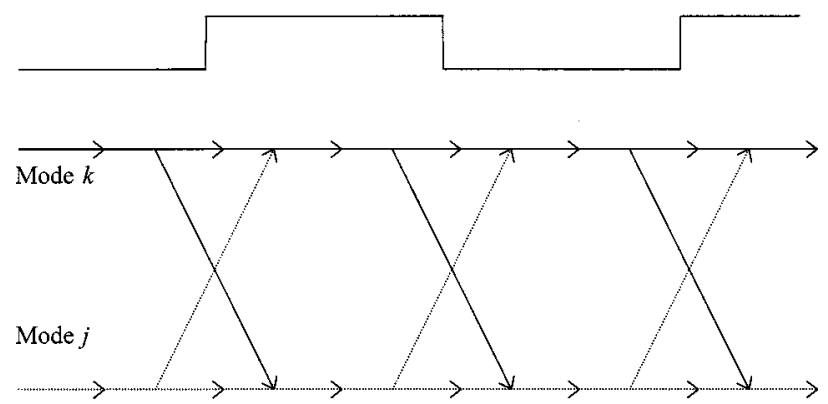

Fig. 5. Schematic diagram showing the mechanism of coupling between two modes in a binary LPG. The couplings occur at the interfaces of regions 1 and 0 with well-defined splitting ratios. The propagation of complex amplitudes of the modes can be described by using transfer matrices. The transfer matrix of a 01 unit cell is composed of four elementary matrices: $\mathbf{F}^{(0 \mid 1)}, \mathbf{P}^{(1)}$, $\mathbf{F}^{(1 \mid 0)}$, and $\mathbf{P}^{(0)}$, which are defined in the text.
The changes in mode amplitudes from region 1 to region 0 are determined by $\overline{\mathbf{A}}=\mathbf{F}^{(0 \mid 1)} \mathbf{A}$ with the following transfer-matrix elements:

$$
\mathrm{F}_{\mathrm{kj}}^{(0 \mid 1)}= \begin{cases}\delta_{\mathrm{j}}=1-\mu^{2} \mathrm{D}_{\mathrm{j}} / 2 & (\mathrm{k}=\mathrm{j}) \\ -\gamma_{\mathrm{kj}}=\mu \frac{-\kappa_{\mathrm{kj}}}{\beta_{\mathrm{k}}^{(0)}-\beta_{\mathrm{j}}^{(0)}} & (\mathrm{k} \neq \mathrm{j})\end{cases}
$$

And the transfer matrices of free propagation through region 0 and region 1 are

$$
\begin{aligned}
& \mathrm{P}_{\mathrm{kj}}^{(0)}=\delta_{\mathrm{kj}} \exp \left[\mathrm{i} \beta_{\mathrm{j}}^{(0)} \Lambda^{(0)}\right], \\
& \mathrm{P}_{\mathrm{kj}}^{(1)}=\delta_{\mathrm{kj}} \exp \left\{\mathrm{i}\left[\beta_{\mathrm{j}}^{(0)}+\mu \beta_{\mathrm{j}}^{(1)}\right] \Lambda^{(1)}\right\} .
\end{aligned}
$$

These matrices will be used in the calculation of the transmission spectra of periodic and quasi-periodic binary LPG's.

$\mathbf{F}=\left[\begin{array}{cc}\exp \left(\mathrm{i} \theta_{\mathrm{k}}\right)\left\{\delta_{\mathrm{k}}^{2}+|\gamma|^{2} \exp \left[\mathrm{i} \Delta^{(1)}\right]\right\} & \exp \left(\mathrm{i} \theta_{\mathrm{k}}\right) \gamma\left\{\delta_{\mathrm{k}}-\delta_{\mathrm{j}} \exp \left[\mathrm{i} \Delta^{(1)}\right]\right\} \\ -\exp \left(\mathrm{i} \theta_{\mathrm{j}}\right) \gamma^{*}\left\{\delta_{\mathrm{j}}-\delta_{\mathrm{k}} \exp \left[-\mathrm{i} \Delta^{(1)}\right]\right\} & \exp \left(\mathrm{i} \theta_{\mathrm{j}}\right)\left\{\delta_{\mathrm{k}}{ }^{2}+|\gamma|^{2} \exp \left[-\mathrm{i} \Delta^{(1)}\right]\right\}\end{array}\right]$

F or the sake of clarity, we have defined the following auxiliary variables:

$$
\theta_{\mathrm{k}}=\theta_{\mathrm{k}}^{(0)}+\theta_{\mathrm{k}}^{(1)}, \quad \theta_{\mathrm{j}}=\theta_{\mathrm{j}}^{(0)}+\theta_{\mathrm{j}}^{(1)},
$$

representing the total increased phase for mode $\mathrm{k}$ and mode j, respectively; and

$$
\Delta^{(1)}=\theta_{\mathrm{j}}^{(1)}-\theta_{\mathrm{k}}^{(1)}, \quad \Delta^{(0)}=\theta_{\mathrm{j}}^{(0)}-\theta_{\mathrm{k}}^{(0)},
$$

representing the phase difference between the two modes within region 1 and region 0 , respectively.

If we neglect the coupling to backward-propagating modes, the forward transfer matrices of multimode couplings can be derived by using Eqs. (15) and (17) in a similar way. We use vector $\mathbf{A}=\left(\mathrm{A}_{0} \mathrm{~A}_{1} \mathrm{~A}_{2} \cdots\right)^{\top}$ to represent the amplitudes of the various modes. The changes in mode amplitudes when the interface from region 0 to region 1 is crossed is then determined by the matrix equation $\mathbf{A}=\mathbf{F}^{(1 \mid 0)} \overline{\mathbf{A}}$, where the elements of the transfer matrix are

$$
\mathrm{F}_{\mathrm{kj}}^{(1 \mid 0)}= \begin{cases}\delta_{\mathrm{j}}=1-\mu^{2} \mathrm{D}_{\mathrm{j}} / 2 & (\mathrm{k}=\mathrm{j}) \\ \gamma_{\mathrm{kj}}=\mu \frac{\kappa_{\mathrm{kj}}}{\beta_{\mathrm{k}}^{(0)}-\beta_{\mathrm{j}}^{(0)}} & (\mathrm{k} \neq \mathrm{j})\end{cases}
$$

\section{TWO-MODE COUPLING IN A PERIODIC GRATING}

In this section we consider two-mode coupling in a periodic LPG by using the forward transfer matrices derived in Section 3. As for the two coupled-mode equations, there is an analytic solution for the transfer-matrix method by using the matrix identities. We will derive this analytic result in this section. The exact solution of two coupled-mode equations with constant coupling coefficients is given in Appendix C. We will compare these two analytic results in specific cases. By using the transfer-matrix method, we can also study the effect of duty cycle on the transmission spectrum of a LPG.

In the case of ideal two-mode coupling, the energy coupled to modes other than these two and to backwardpropagating modes when the heterointerface is crossed are neglected. Since the structure under study has timereversal symmetry, then, with a properly chosen phase origin, the parameters $\gamma$ and $\delta$ in the following can be taken as real. The power conservation condition then requires that

$$
\delta_{\mathrm{k}}=\delta_{\mathrm{j}}=\sqrt{1-\gamma^{2}} \equiv \delta .
$$

Thus the unit transfer matrix in the ideal case is

$$
\mathbf{F}=\exp (\mathrm{i} \bar{\theta})\left[\begin{array}{cc}
\exp \left(\mathrm{i} \frac{-\Delta}{2}\right)\left\{\delta^{2}+\gamma^{2} \exp \left[\mathrm{i} \Delta^{(1)}\right]\right\} & \exp \left(\mathrm{i} \frac{-\Delta}{2}\right) \gamma \delta\left\{1-\exp \left[\mathrm{i} \Delta^{(1)}\right]\right\} \\
-\exp \left(\mathrm{i} \frac{\Delta}{2}\right) \gamma \delta\left\{1-\exp \left[-\mathrm{i} \Delta^{(1)}\right]\right\} & \exp \left(\mathrm{i} \frac{\Delta}{2}\right)\left\{\delta^{2}+\gamma^{2} \exp \left[-\mathrm{i} \Delta^{(1)}\right]\right\}
\end{array}\right] \equiv \exp (\mathrm{i} \bar{\theta})\left[\begin{array}{ll}
\mathrm{A} & \mathrm{B} \\
\mathrm{C} & \mathrm{D}
\end{array}\right],
$$


where $\bar{\theta} \equiv\left(\theta_{\mathrm{k}}+\theta_{\mathrm{j}}\right) / 2$ is the average phase shift and $\Delta$ $=\Delta^{(1)}+\Delta^{(2)}$ is the total phase difference. We can prove that the unit transfer matrix satisfies the unitary condition:

$$
\mathbf{F}^{+} \mathbf{F}=\mathbf{I} .
$$

Using the well-known Chebyshev identity, ${ }^{10}$ we have the following expression for the total $\mathrm{N}$-period transfer matrix:

$$
\kappa_{\mathrm{kj}} \cong \frac{\omega \epsilon_{0} \mathrm{n}_{1}}{2} \Delta \mathrm{n}_{\mathrm{g}} \int_{\mathrm{A}_{\mathrm{co}}}\left(\mathbf{e}_{\mathrm{t} 01}^{\mathrm{co}} * \cdot \mathbf{e}_{\mathrm{t} 1 \nu}^{\mathrm{d}}\right) \mathrm{dA}=\kappa_{01-1 \nu}^{\mathrm{co-c}},
$$

where $\Delta \mathrm{n}_{\mathrm{g}}$ is the photoinduced index increase in region 1 and is assumed to be $5 \times 10^{-4}$ in the following calculation. The grating period is chosen to be $500 \mu \mathrm{m}$ in the following simulation. From the phase-matching condition (43) with the aid of the calculated dispersion relation, i.e., $\beta$ as a function of $\lambda$, the resonance wavelength is
$\mathbf{F}^{N}=\exp (\mathrm{iN} \bar{\theta})\left[\begin{array}{c}\frac{\mathrm{A} \sin (\mathrm{NK} \Lambda)-\sin [(\mathrm{N}-1) \mathrm{K} \Lambda]}{\sin (\mathrm{K} \Lambda)} \\ \mathrm{C} \frac{\sin (\mathrm{NK} \Lambda)}{\sin (\mathrm{K} \Lambda)}\end{array}\right.$

$\frac{B \frac{\sin (N K \Lambda)}{\sin (K \Lambda)}}{D \sin (N K \Lambda)-\sin [(N-1) K \Lambda]} \quad$ where the parameter $\mathrm{K} \Lambda$ is given by

$$
\begin{aligned}
\cos (\mathrm{K} \Lambda)= & \frac{1}{2}(\mathrm{~A}+\mathrm{D})=\left(1-\gamma^{2}\right) \cos \left[\frac{\Delta^{(1)}+\Delta^{(0)}}{2}\right] \\
& +\gamma^{2} \cos \left[\frac{\Delta^{(1)}-\Delta^{(0)}}{2}\right] .
\end{aligned}
$$

It is seen from Eq. (37) that for coherent coupling between the two modes, the parameter $\mathrm{K} \Lambda$ is required to approach $\pm m \pi$, where $m$ is an integer, which results in the following coherent coupling condition:

$$
\Delta^{(1)}+\Delta^{(0)} \cong \pm 2 \mathrm{~m} \pi .
$$

Note that the above expression is valid when the parameter $\gamma$ is small compared with unity. By substituting Eqs. (33) into relation (42), we obtain the following equation, which corresponds to the first Fourier component of the grating [here we assume that $\beta_{\mathrm{k}}^{(0)}>\beta_{\mathrm{j}}^{(0)}$, so we choose $-2 \pi$ for $\left.\Delta^{(1)}+\Delta^{(0)}\right]$ :

$$
\left[\beta_{\mathrm{k}}^{(0)}-\beta_{\mathrm{j}}^{(0)}-\frac{2 \pi}{\Lambda}\right]+\mu\left[\beta_{\mathrm{k}}^{(1)}-\beta_{\mathrm{j}}^{(1)}\right] \frac{\Lambda^{(1)}}{\Lambda}=0,
$$

in which the zero order is the well-known phase-matching condition. Now let us apply the above formula to the coupling of a core mode and a specific cladding mode of a single-mode fiber. Note that the perturbation expansion parameter $\mu$ will be set to unity from now on. The calculation of propagation constants and coupling coefficients of a three-layered fiber waveguide can be found in Ref. 7. The same parameters as those described in Ref. 7 are chosen in the following calculations: $n_{1}=1.458$ is the index of the core region, $\Delta=\left(n_{1}-n_{2}\right) / n_{1}=0.0055$ is the normalized core-cladding index difference, $a_{1}$ $=2.625 \mu \mathrm{m}$ is the core radius, and $\mathrm{a}_{2}=62.5 \mu \mathrm{m}$ is the cladding radius. Let mode $k$ be the core mode $\left(\mathrm{LP}_{01}\right)$ and mode $\mathrm{j}$ be the ninth cladding mode $\left(\mathrm{HE}_{1 \nu}\right.$, with $\left.\nu=9\right)$. As mentioned in Ref. 7, the z-component coupling coefficient is much smaller than the transverse coefficients. So we consider only the transverse coupling coefficients. We therefore have in this case found to be approximately $1820 \mathrm{~nm}$. The calculated coupling coefficient at this wavelength divided by $\Delta \mathrm{n}_{\mathrm{g}}$ for $\nu$ $=9$ is $\kappa_{01-1 \nu}^{\mathrm{coc}} / \Delta \mathrm{n}_{\mathrm{g}}=0.351$. The propagation constants of the two coupled modes at the resonance wavelength are $\beta_{\mathrm{k}}^{(0)}=\beta_{01}^{\mathrm{co}}=2 \pi \mathrm{n}_{01}^{\mathrm{co}} / \lambda$ and $\beta_{\mathrm{j}}^{(0)}=\beta_{1 v}^{\mathrm{cl}}=2 \pi \mathrm{n}_{1 v}^{\mathrm{c}} / \lambda$, where $\lambda$ is the free-space wavelength, and the effective indices for the two modes are found to be $\mathrm{n}_{01}^{\mathrm{co}}=1.4514$ and $\mathrm{n}_{1 \nu}^{\mathrm{d}}$ $=1.4479$ for $\nu=9$. We neglect the waveguide dispersion in the vicinity of the resonant wavelength. In Section 5 we will take into account the effect of waveguide dispersion in the calculation of multimode coupling. The first-order correction of propagation constants in region 1 is in this case [cf. Eqs. (19) and (A5)]

$$
\beta_{\mathrm{k}}^{(1)} \cong \frac{\omega \epsilon_{0} \mathrm{n}_{1}}{2} \Delta \mathrm{n}_{\mathrm{g}} \int_{\mathrm{A}_{\mathrm{co}}}\left(\mathbf{e}_{\mathrm{t} 01}^{\mathrm{co}} * \cdot \mathbf{e}_{\mathrm{t} 01}^{\mathrm{co}}\right) \mathrm{dA}=\kappa_{01-01}^{\mathrm{co}-\mathrm{co}},
$$

and we will neglect the correction of the propagation constant of the cladding mode, ${ }^{7}$ which results in $\beta_{j}^{(1)}=0$. The calculated self-coupling divided by $\Delta \mathrm{n}_{\mathrm{g}}$ of the core mode is $\kappa_{01-01}^{\mathrm{CO}-\mathrm{CO}} / \Delta \mathrm{n}_{\mathrm{g}}=1.5576$.

In Fig. 6 we show the transmission spectra of binary LPG's with different numbers of unit cells. The transmission loss is seen to increase with the number of unit cells. As the number of cells further increases, however, the transmission loss then decreases to zero again, as

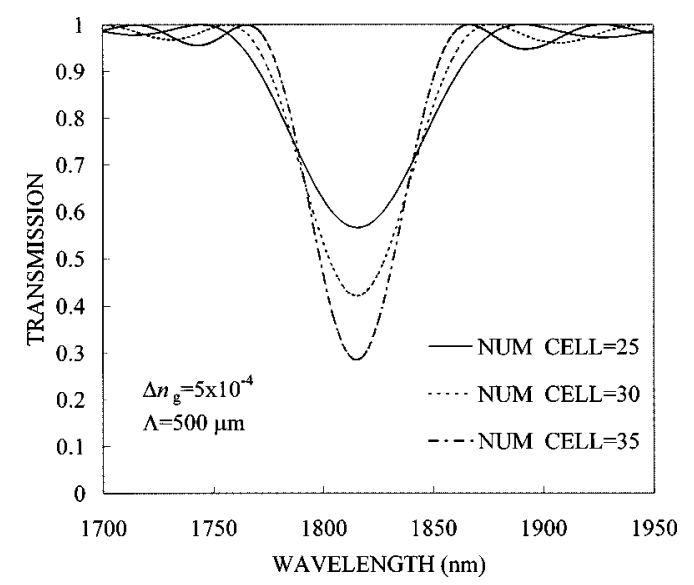

Fig. 6. Comparison of transmission spectra for binary LPG's with number of cells equal to 25,30 , and 35 . 


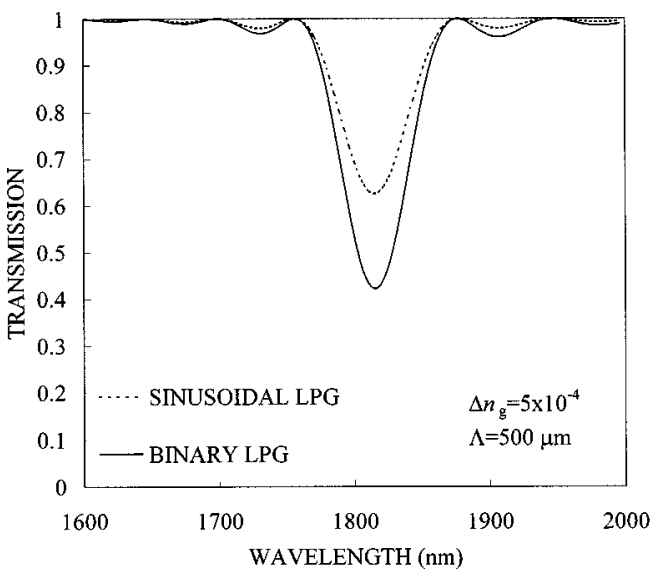

(a)

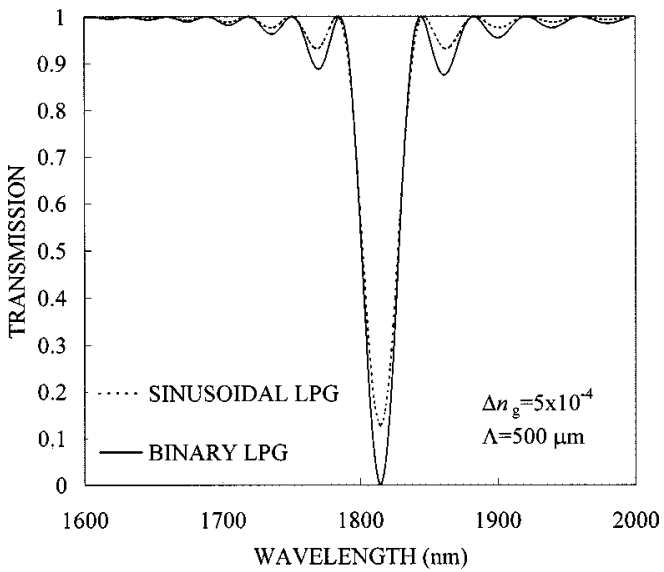

(b)

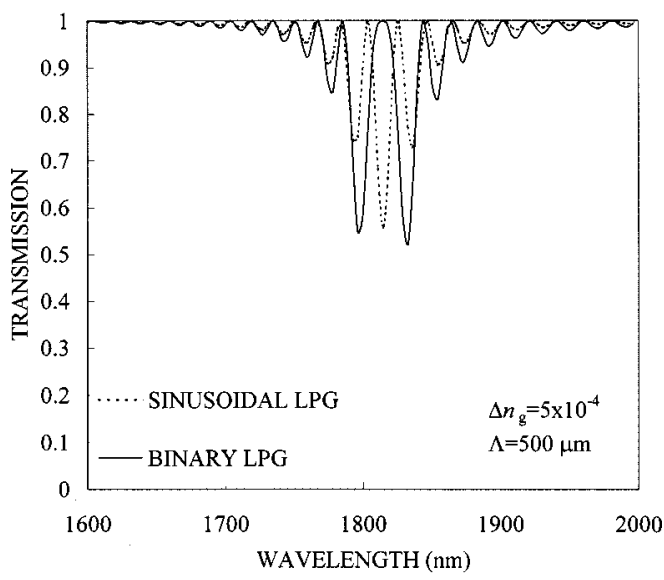

(c)

Fig. 7. Transmission spectra of a binary and a uniform sinusoidal LPG. Each has a grating length of (a) $15 \mathrm{~mm}$, (b) 27.5 $\mathrm{mm}$, and (c) $55 \mathrm{~mm}$.

shown in Fig. 7. This is because the power coupled to the cladding mode is coupled back to the core mode. In Fig. 7 we also compare the transmission spectra of the binary LPG's using our model with those of uniform sinusoidal LPG's for different numbers of unit cells (i.e., different grating lengths). The parameters are adjusted to be the same. For comparison, the duty cycle is set to unity in the case of binary LPG's. Note that there is an analytic solution for the uniform sinusoidal LPG with two-mode coupling that is based on the coupled-mode theory. We briefly describe the results in Appendix C. In Fig. 7(a) the number of unit cells is 30 in a binary $L P G\left(L_{g}\right.$ $=15 \mathrm{~mm}$ ). At this grating length, the transmission loss of the binary LPG with duty cycle equal to unity is larger than that of a uniform sinusoidal LPG. This can be explained as follows. Since the grating strength is the same in both cases $\left(\Delta \mathrm{n}_{\mathrm{g}}=5 \times 10^{-4}\right)$, the Fourier-series expansions of the two gratings are as follows:

$$
\begin{aligned}
\Delta \mathrm{n}_{\sin }(\mathrm{z}) & =\Delta \mathrm{n}_{\mathrm{g}} \frac{1}{2}[1+\sin (K z)] \\
& =\Delta \mathrm{n}_{\mathrm{g}}\left[\frac{1}{2}+\frac{1}{4 \mathrm{i}} \exp (\mathrm{iKz})-\frac{1}{4 \mathrm{i}} \exp (-\mathrm{iKz})\right],
\end{aligned}
$$

$$
\begin{aligned}
\Delta \mathrm{n}_{\text {bin }}(\mathrm{z})= & \Delta \mathrm{n}_{\mathrm{g}} \sum_{\mathrm{n}}\left[\mathrm{u}(\mathrm{z}-\mathrm{n} \Lambda)-\mathrm{u}\left(\mathrm{z}-\mathrm{n} \Lambda-\Lambda^{(1)}\right)\right] \\
= & \Delta \mathrm{n}_{\mathrm{g}}\left[\frac{1}{2}+\frac{1}{\pi \mathrm{i}} \exp (\mathrm{i} K z)-\frac{1}{\pi \mathrm{i}} \exp (-\mathrm{i} K z)\right. \\
& \left.+\frac{1}{3 \pi \mathrm{i}} \exp (\mathrm{i} 3 K z)-\frac{1}{3 \pi \mathrm{i}} \exp (-\mathrm{i} 3 K z)+\cdots\right],
\end{aligned}
$$

where $K=2 \pi / \Lambda$. We can see that the magnitude of the first-order Fourier component $[\exp (\mathrm{iKz})]$ of a binary LPG is $4 / \pi$ larger than that of a sinusoidal LPG.

Figure 7(b) shows the saturation of transmission loss of a binary LPG. The number of unit cells is 55 in this case $\left(\mathrm{L}_{\mathrm{g}}=27.5 \mathrm{~mm}\right)$. In Fig. 7(c) the grating length further increases to $55 \mathrm{~mm}$. Note that the original transmission dips of both gratings shrink toward zero. This is because the LPG is, in fact, a codirectional coupler, so as the length is further increased, the energy will again couple back to the core mode.

The effect of duty cycle on the transmission spectrum of a binary LPG is shown in Fig. 8. The duty cycle is defined as the ratio of the length of region 1 to that of region 0 within a period. We designate the ratio as $R$ $\equiv \Lambda^{(1)} / \Lambda^{(0)}$. From Eq. (43) and relation (45), it can be deduced that if the waveguide dispersion is not included,

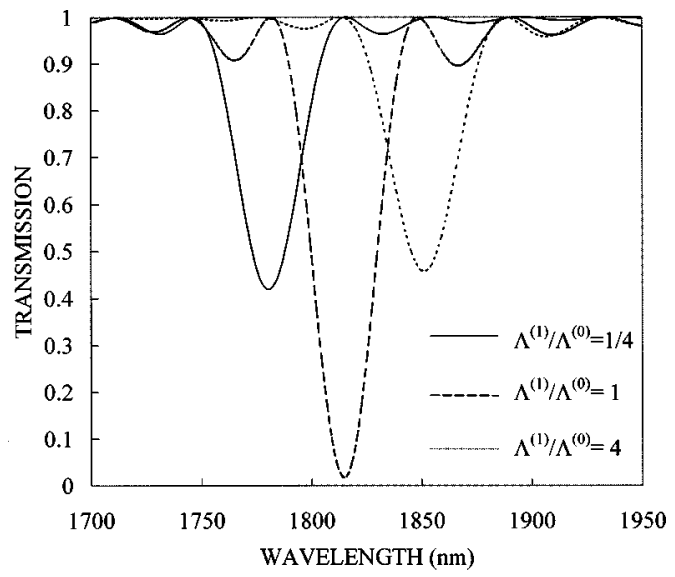

Fig. 8. Comparison of transmission spectra for binary LPG's with different duty cycles. 
the center wavelength (wavelength with largest transmission loss) $\lambda_{0}$ is determined by the following expression:

$$
\lambda_{0}=\left(\mathrm{n}_{01}^{\mathrm{co}}-\mathrm{n}_{1 v}^{\mathrm{d}}\right) \Lambda /\left(1-\frac{\kappa_{01-01}^{\mathrm{co}-\mathrm{CO}} \Lambda}{2 \pi} \frac{\mathrm{R}}{1+\mathrm{R}}\right) .
$$

As the ratio $R$ increases, the center wavelength shifts to the larger one as shown in Fig. 8. The transmission loss also varies with duty cycle and reaches a maximum when the duty cycle is unity.

\section{TRANSMISSION SPECTRUM OF A PERIODIC BINARY LONG-PERIOD GRATING}

Now we apply the transfer-matrix method to describe the evolution of mode amplitudes in a periodic binary LPG. The modes involved in the couplings of a LPG include the fundamental core mode $L_{01}$ and the cladding modes. The symbols used here to label the quantities relevant to cladding modes are consistent with those used in Ref. 7. The cladding modes are hybrid modes in a cylindrical waveguide and are labeled by using the azimuthal orders I and $\nu$, which are called the cladding-mode numbers. Since the fundamental core mode has azimuthal number $\mathrm{I}=1$, the cladding modes with nonvanishing coupling coefficients are those with the same azimuthal order as that of the core mode, i.e., I = 1 . The forward transfer matrices of the two interfaces can be symbolically written as follows (only the relevant matrix elements are listed): for incidence from region 0 to region 1 :

$$
\left(\begin{array}{c}
\mathrm{A}^{\mathrm{co}} \\
\mathrm{A}_{1 \nu}^{\mathrm{cl}}
\end{array}\right)=\left[\begin{array}{cc}
1-\frac{\mathrm{D}_{01}^{\mathrm{co}}}{2} & \frac{\kappa_{01-1 \nu}^{\mathrm{co}-\mathrm{d}}}{\beta_{01}^{\mathrm{co}}-\beta_{1 \nu}^{\mathrm{cl}}} \\
\kappa_{1 \nu}^{\mathrm{cl}-0} & 1-\frac{\mathrm{D}_{1 \nu}^{\mathrm{c}}}{2}
\end{array}\right]\left(\begin{array}{l}
\overline{\mathrm{A}}^{\mathrm{co}} \\
\overline{\mathrm{A}}_{1 \nu}^{\mathrm{cl}}
\end{array}\right) \equiv \mathbf{F}^{(1 \mid 0)}\left(\begin{array}{l}
\overline{\mathrm{A}}_{1 \nu}^{\mathrm{co}} \\
\overline{\mathrm{A}}_{1 \nu}^{\mathrm{cl}}-\beta_{01}^{\mathrm{co}}
\end{array}\right) ;
$$

and for incidence from region 1 to region 0 :

$$
\left(\begin{array}{l}
\overline{\mathrm{A}}^{\mathrm{co}} \\
\overline{\mathrm{A}}_{1 \nu}^{\mathrm{d}}
\end{array}\right)=\left[\begin{array}{cc}
1-\frac{\mathrm{D}_{01}^{\mathrm{co}}}{2} & \frac{-\kappa_{01-1 \nu}^{\mathrm{co}-\mathrm{cl}}}{\beta_{01}^{\mathrm{co}}-\beta_{1 \nu}^{\mathrm{cl}}} \\
-\kappa_{1 \nu-01}^{\mathrm{c}-\mathrm{co}} & 1-\frac{\mathrm{D}_{1 \nu}^{\mathrm{cl}}}{2}
\end{array}\right]\left(\begin{array}{c}
\mathrm{A}^{\mathrm{co}} \\
\mathrm{A}_{1 \nu}^{\mathrm{cl}}
\end{array}\right) \equiv \mathbf{F}^{(0 \mid 1)}\left(\begin{array}{c}
\mathrm{A}^{\mathrm{co}} \\
\mathrm{A}_{1 \nu}^{\mathrm{cl}}
\end{array}\right),
$$

where $\nu=1,2,3, \ldots$ is the mode number of cladding modes. We also neglect the couplings among different cladding modes. ${ }^{7}$ In the calculations of parameters $D_{01}^{\text {co }}$ and $D_{1 v}^{\mathrm{cl}}$ with the use of $\mathrm{Eq}$. (A17), we may neglect the contribution from the reflection part. The transfer matrices for free propagation are given below:

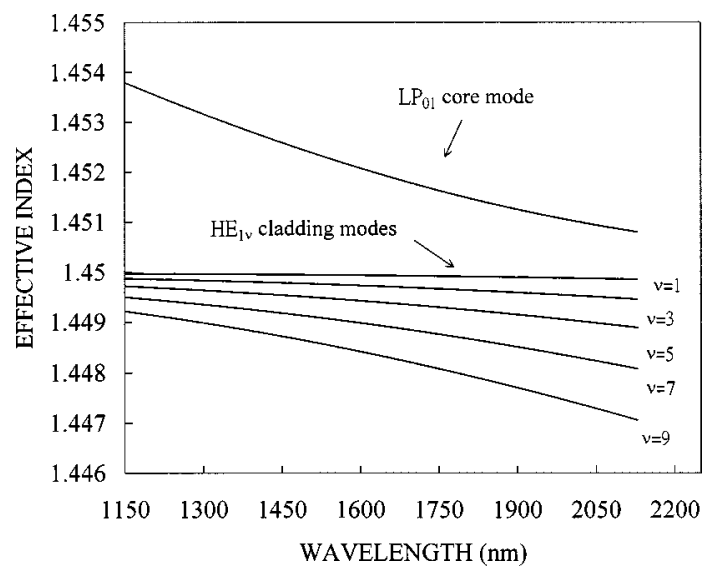

Fig. 9. Dispersion curves of effective indices of $L P_{01}$ core mode and $\mathrm{HE}_{1 \nu}$ cladding modes with $\nu=1,3,5,7,9$.

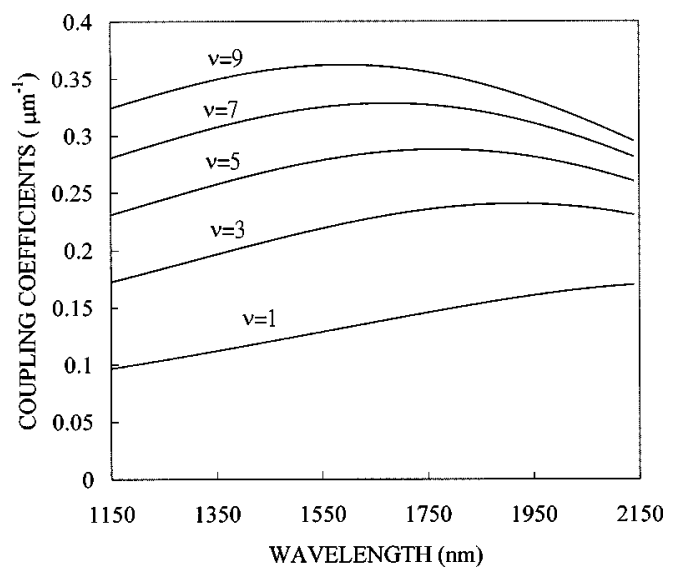

Fig. 10. Dispersion curves of coupling coefficients $\kappa_{01-1 \nu}^{\mathrm{co}-\mathrm{cl}}$ of cladding modes with $\nu=1,3,5,7,9$.

Then the propagation of mode amplitudes by using the unit transfer matrix $\mathbf{F}=\mathbf{P}^{(0)} \mathbf{F}^{(0 \mid 1)} \mathbf{P}^{(1)} \mathbf{F}^{(1 \mid 0)}$ is described as follows:

$$
\left(\begin{array}{l}
A^{c o}(n+1) \\
A_{1}^{d}(n+1) \\
A_{2}^{d}(n+1) \\
\vdots
\end{array}\right)=\mathbf{F}\left(\begin{array}{l}
A^{c o}(n) \\
A_{1}^{c l}(n) \\
A_{2}^{c l}(n) \\
\vdots
\end{array}\right) .
$$

In the following calculation of the transmission spectrum, we take into account the effect of waveguide dispersion, namely, the wavelength dependence of propagation constants (or effective indices) and coupling coefficients. The calculation of mode properties of a threelayered fiber waveguide can be found in Ref. 7. The parameters of the three-layered fiber used here are described in Section 4.

$$
\begin{aligned}
& \mathbf{P}^{(0)}=\left[\begin{array}{cc}
\exp \left[\mathrm{i} \beta_{01}^{\mathrm{co}} \Lambda^{(0)}\right] & 0 \\
0 & \exp \left[\mathrm{i} \beta_{1 \nu}^{\mathrm{dl}} \Lambda^{(0)}\right]
\end{array}\right], \\
& \mathbf{P}^{(1)}=\left[\begin{array}{cc}
\exp \left[\mathrm{i}\left(\beta_{01}^{\mathrm{co}}+\kappa_{01-01}^{\mathrm{co}-\mathrm{co}}\right) \Lambda^{(1)}\right] & 0 \\
0 & \exp \left[\mathrm{i}\left(\beta_{1 \nu}^{\mathrm{cl}}+\kappa_{1 \nu-1 \nu}^{\mathrm{d}-\mathrm{cl}}\right) \Lambda^{(1)}\right]
\end{array}\right] .
\end{aligned}
$$


In Fig. 9 we show the dependence of effective indices on wavelength for odd cladding modes and core mode. We can see from the figure that the dispersion is more severe for higher-order cladding modes. In general, effective index decreases with wavelength, as shown in the figure. However, the dispersion of the core mode is different from that of the cladding modes in that the dispersion curve of the core mode is concave while those of the cladding modes are convex. In Fig. 10 we show the dependence of

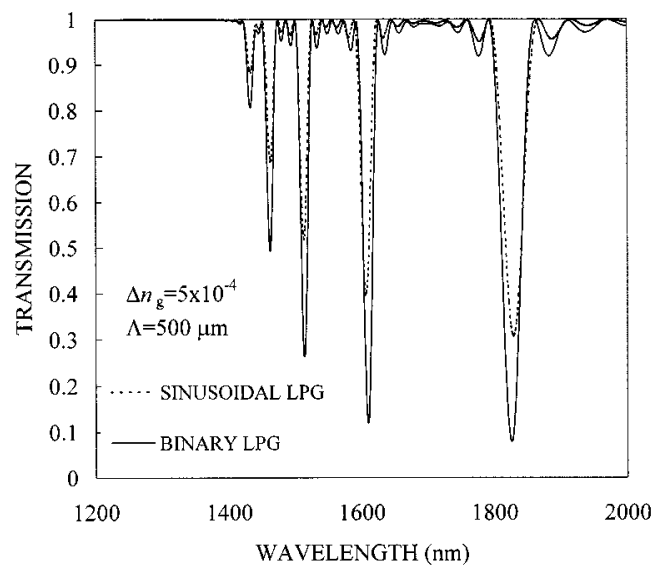

(a)

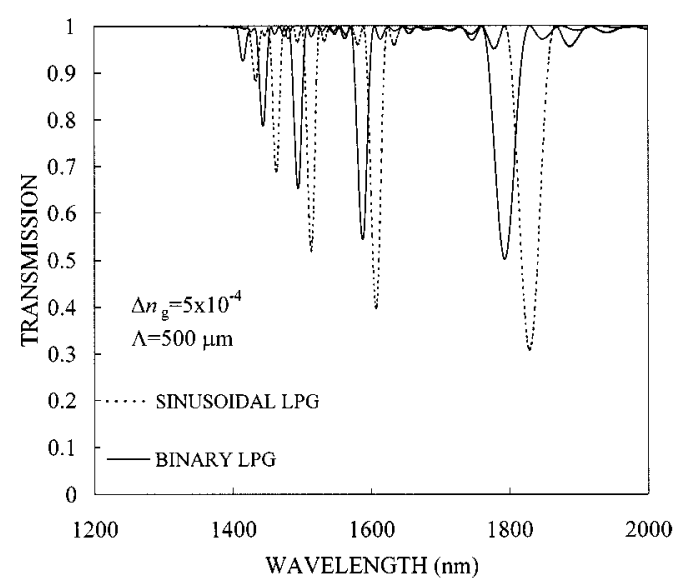

(b)

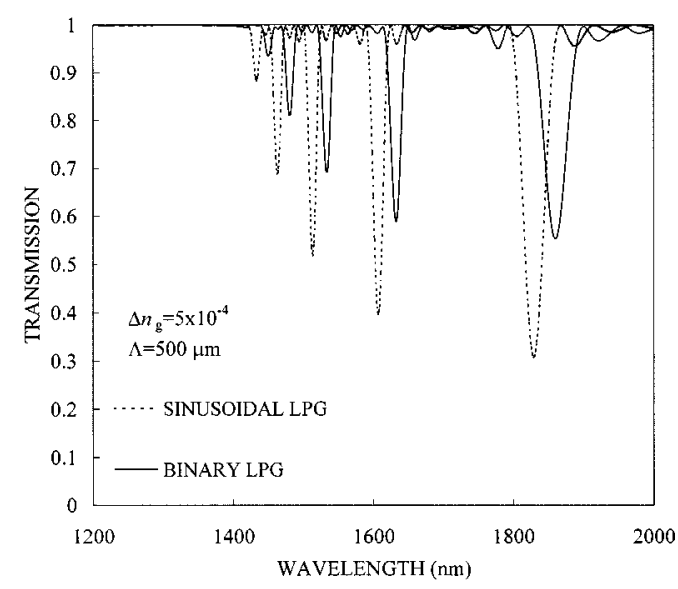

(c)

Fig. 11. Transmission spectra of a binary and a uniform sinusoidal LPG. The duty cycle of the binary LPG is set to (a) unity, (b) $1 / 4$, and (c) 4 . coupling coefficients $\kappa_{01-1 \nu}^{\mathrm{co}-\mathrm{cl}}$ on wavelength for cladding modes with mode numbers $\nu=1,3,5,7,9$. Take cladding mode $\nu=9$ as an example; the variation of the coupling coefficient has a convex shape and reaches a maximum at wavelength $\approx 1550 \mathrm{~nm}$.

The transmission spectrum of a periodic binary LPG with multimode coupling is shown in Fig. 11(a). Also shown is the spectrum of a uniform sinusoidal LPG obtained by using the coupled-mode theory for comparison. The coupled-mode theory for the transmission spectrum of a periodic LPG can be found in Ref. 7 and is highlighted in Appendix C. In the wavelength range shown, we consider couplings of the core mode with five odd cladding modes. The grating strength $\Delta \mathrm{n}_{\mathrm{g}}$ is $5 \times 10^{-4}$, and the grating period is $500 \mu \mathrm{m}$. The duty cycle is set to unity, and the number of cells is 45, which corresponds to 90 heterolayers. As explained in Section 4, the transmission loss of a binary LPG is larger than that of a uniform sinusoidal LPG. The transmission loss increases with the order of the cladding modes in this grating length. When the grating length further increases, some transmission dips will shrink back toward zero, as discussed in Section 4.

The effect of duty cycle $R=\Lambda^{(1)} / \Lambda^{(0)}$ on the transmission spectra is shown in Figs. 11 (b) and 11 (c). In Fig. 11 (b) the duty cycle is set to $1 / 4$. The transmission dips shift toward shorter wavelengths, which can be explained by using the resonance condition (43), from which the cause for the shifts is the correction of the propagation constant of the core mode in region 1 . The case of duty cycle equal to 4 is shown in Fig. 11(c). From these plots we conclude that the transmission loss of a periodic binary LPG reaches a maximum when the duty cycle is unity.

\section{TRANSMISSION SPECTRUM OF A QUASI-PERIODIC LONG-PERIOD GRATING}

To demonstrate the usefulness of the transfer-matrix method, we apply it to the study of the transmission spectrum of a quasi-periodic structure. The structure definition of quasi-periodic LPG's will be given first. Then we will discuss the resonance conditions for a quasi-periodic LPG. The structure factor of a LPG will be introduced. Using the resonance conditions, we can determine the location of transmission dips. Finally, we will study a Fibonacci layered binary LPG and discuss its transmission spectrum.

For a quasi-periodic LPG, we first determine the Bravis lattice points ${ }^{13,14}$ of the quasi-periodic structure, $\left\{z_{0}, z_{1}\right.$, $\left.z_{2}, \ldots\right\}$, and then place a region 1 with length $\Lambda^{(1)}$ at each lattice point. The result is schematically shown in Fig. 12. Having defined the grating pattern, we can use the following method to find the resonance condition. Consider the weak-coupling limit, or the nondepletion limit, which means that the amplitude of the core mode remains almost unchanged after a region 1 is propagated. Each region 1 acts as a diffractor that couples energy from core mode to cladding mode. Let $\eta$ be the amplitude splitting ratio from a core mode to a specific cladding mode through the diffractor, e.g., mode $\nu$. The total transmitted amplitude of the cladding mode is then the contribution of all 


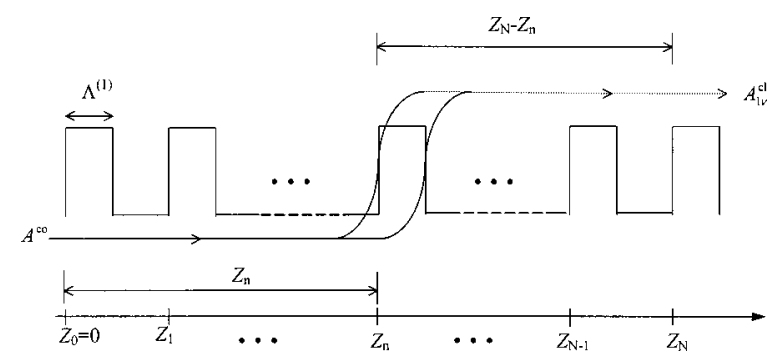

Fig. 12. Schematic diagram of a quasi-periodic binary grating. The point set $\left\{z_{0}, z_{1}, z_{2}, \ldots\right\}$ forms the Bravis lattice of the quasi-periodic lattice. Each lattice point is then placed in a region 1 , which acts as a diffractor.

diffractors. Let the total number of lattice points be $\mathrm{N}$; the contribution of the path through the $\mathrm{nth}$ diffractor has the phase $\exp \left[\mathrm{i} \beta_{01}^{\mathrm{co}} z_{\mathrm{n}}+\mathrm{in} \kappa_{01-01}^{\mathrm{co}-\mathrm{co}} \Lambda^{(1)}\right] \eta$ $\times \exp \left[i \beta_{1 v}^{\mathrm{cl}}\left(\mathrm{z}_{\mathrm{N}}-\mathrm{z}_{\mathrm{n}}\right)\right]$, where we have set $\mathrm{z}_{0}=0$ (cf. Fig. 12). We obtain the following total transmission amplitude of the $\nu$ th cladding mode:

$$
\begin{aligned}
\mathrm{A}_{1 \nu}^{\mathrm{cl}} \cong & \mathrm{A}^{\mathrm{co}}(0) \exp \left(\mathrm{i} \beta_{1_{\nu}}^{\mathrm{cl}} \mathrm{z}_{\mathrm{N}}\right) \eta \sum_{\mathrm{n}=0}^{\mathrm{N}} \exp \left\{\mathrm { i } \left[\left(\beta_{01}^{\mathrm{co}}-\beta_{1_{\nu}}^{\mathrm{cl}}\right) \mathrm{z}_{\mathrm{n}}\right.\right. \\
& \left.\left.+\mathrm{n} \kappa_{01-01}^{\mathrm{co}-\mathrm{co}} \Lambda^{(1)}\right]\right\} \\
\propto & \eta \sum_{\mathrm{n}=1}^{\mathrm{N}} \exp \left[\mathrm{iQ} \mathrm{z}_{\mathrm{n}}+\mathrm{n} \kappa_{01-01}^{\mathrm{co}-\mathrm{co}} \Lambda^{(1)}\right] \equiv \eta \mathrm{S}(\mathrm{Q}), \quad
\end{aligned}
$$

where we have defined the structure factor $\mathrm{S}(\mathrm{Q})$ and $\mathrm{Q}$ $\equiv \beta_{01}^{\mathrm{CO}}-\beta_{1 \nu}^{\mathrm{cl}}$. The resonant wavel engths are then determined from the structure factor. For example, the structure factor of an infinite periodic LPG is $S(Q)$ $=(2 \pi / \Lambda) \Sigma_{\mathrm{n}} \delta\left(\mathrm{Q}+\kappa_{01-01}^{\mathrm{coc}-\mathrm{co}} / 2-\mathrm{n} 2 \pi / \Lambda\right)$, consisting of many delta peaks. For the peak $n=1$, we obtain the usual resonance condition (43).

We now study a special case of quasi-periodic structure, the Fibonacci lattice. The Bravis lattice point of a $\mathrm{Fi}$ bonacci lattice can be expressed as ${ }^{15}$

$$
\left.\mathrm{z}_{\mathrm{n}}=\Lambda_{\mathrm{B}}\left(\mathrm{n}+\frac{1}{\tau^{\prime}} \mid \frac{\mathrm{n}}{\tau}\right]\right),
$$

where $\tau$ is the "golden mean" $[(1+\sqrt{5}) / 2]$ and $\tau^{\prime}$ is a parameter to be explained below. The spacing of two successive points $z_{n+1}-z_{n}$ is either $\Lambda_{B}$ or $\Lambda_{A}=(1$ $\left.+1 / \tau^{\prime}\right) \Lambda_{\mathrm{B}}$, i.e., $\left(1+1 / \tau^{\prime}\right)$ is the ratio of the two lattice spacings. I] represents the greatest-integer function. This lattice has been shown to be quasi-periodic with two linearly independent periods of ratio equal to $\tau$. Let $\kappa_{01-01}^{\mathrm{co}-\mathrm{Co}} \Lambda^{(1)} / \mathrm{Q} \Lambda_{\mathrm{B}}=\alpha$; then the structure factor of the $\mathrm{Fi}$ bonacci LPG is

$$
\mathrm{S}(\mathrm{Q})=\sum_{\mathrm{n}} \exp \left\{\mathrm{iQ}\left[\mathrm{n}(1+\alpha)+\frac{1}{\tau^{\prime}}\left|\frac{\mathrm{n}}{\tau}\right|\right] \Lambda_{\mathrm{B}}\right\} .
$$

For an infinitely long Fibonacci lattice, it can be shown that the structure factor above has the following form ${ }^{15}$ :

$$
\mathrm{S}(\mathrm{Q}) \propto \sum_{\mathrm{p}, \mathrm{q}} \frac{\sin \mathrm{X}_{\mathrm{p}, \mathrm{q}}}{\mathrm{X}_{\mathrm{p}, \mathrm{q}}} \exp \left(\mathrm{i} \mathrm{X}_{\mathrm{p}, \mathrm{q}}\right) \delta\left(\mathrm{Q}-\mathrm{Q}_{\mathrm{p}, \mathrm{q}}\right),
$$

where

$$
\mathrm{Q}_{\mathrm{p}, \mathrm{q}}=\frac{\tau^{\prime}(\mathrm{q}+\mathrm{p} \tau)}{1+\tau^{\prime} \tau(1+\alpha)} \frac{2 \pi}{\Lambda_{\mathrm{B}}}
$$

and $X_{p, q}=2 \pi q-\Lambda_{B} Q_{p, q} / \tau^{\prime}$. Thus the constructive addition of the diffracted waves occurs when $Q=Q_{p, q}$, which is the resonance condition for a Fibonacci LPG. The prefactor of the delta function determines the relative magnitude of the resonant peaks. ${ }^{14,15}$ If we neglect the correction term $\alpha \Lambda_{\mathrm{B}}=\kappa_{01-01}^{\mathrm{co}-\mathrm{co}} \Lambda^{(1)}$ and let $\tau^{\prime}=\tau$, then the resonance condition can be simplified to the following form:

$$
\beta_{01}^{\mathrm{co}}-\beta_{1_{\nu}}^{\mathrm{cl}}-(\mathrm{m}+\mathrm{n} \tau) \frac{2 \pi}{\bar{\Lambda}}=0
$$

where we have used $\tau^{2}=\tau+1$ and $\bar{\Lambda}=\tau \Lambda_{\mathrm{A}}+\Lambda_{\mathrm{B}}$ is the average period of the lattice. Thus, for a specific cladding mode, say mode $\nu$, we will use the notation $\lambda_{\nu}^{(m, n)}$ or just $(m, n)$ to represent the resonance wavelength.

Using the elementary transfer matrices $\mathbf{F}^{(0 \mid 1)}, \mathbf{F}^{(1 \mid 0)}$, $\mathbf{P}^{(0)}$, and $\mathbf{P}^{(1)}$ described in Section 5, we can calculate the transmission spectrum of a Fibonacci LPG, which is shown in Fig. 13. The grating strength $\Delta \mathrm{n}_{\mathrm{g}}$ is 3.5 $\times 10^{-4}$. The average grating period $\bar{\Lambda}$ is $1200 \mu \mathrm{m}$. Additionally, the parameter $\tau^{\prime}$ is set to the golden mean, so we can use Eq. (59) to estimate the location of transmission dips. In Fig. 13 we also label the different dips with the cladding-mode number that contributes to this transmission loss. There are several features worth noting. First, for a specific cladding mode, the constructive coupling of energy from the core mode occurs at several wavel ength ranges, while in a periodic LPG, the constructive coupling wavelength range is unity for each cladding mode for the first harmonic of the grating. In other words, a single cladding mode will contribute to several transmission loss dips. Second, the transmission dips are distributed approximately into three groups in this wavelength range. We use arrows of different styles to indicate the corresponding groups. This phenomenon can be explained by using the resonance condition (59). For example, Fig. 14 shows the transmission spectrum of the $\nu=3$ cladding mode, and we use the label $(\mathrm{m}, \mathrm{n})$ to

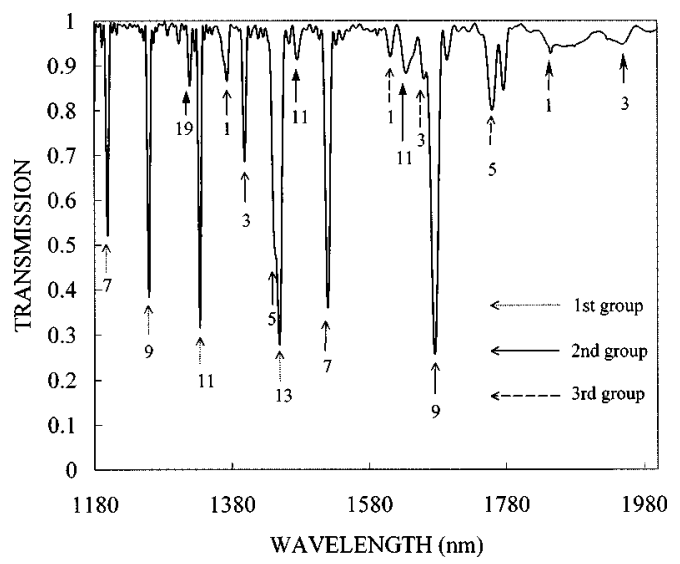

Fig. 13. Transmission spectrum of a quasi-periodic Fibonacci LPG. The transmission dips are grouped according to the resonance conditions $(m, n)$. The first group corresponds to $(1,2)$, the second corresponds to $(1,1)$, and the third corresponds to $(0,1)$. 


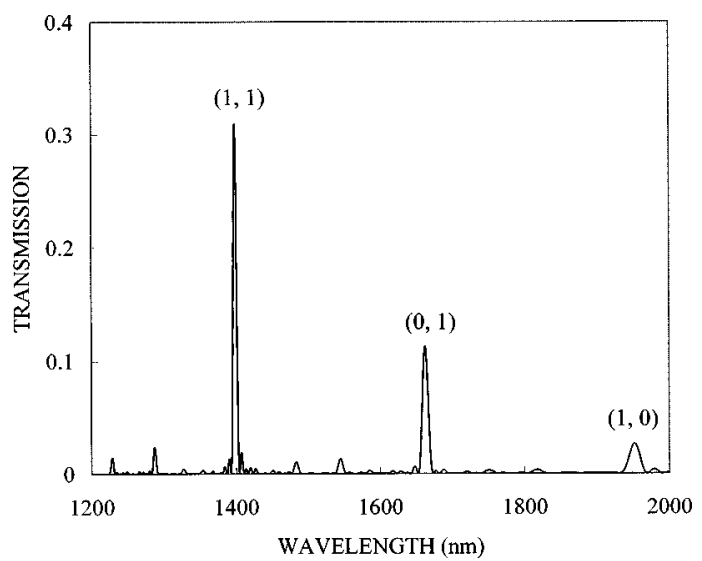

Fig. 14. Transmission spectrum of the $\nu=3$ cladding mode in a quasi-periodic Fibonacci LPG. The labels above the main peaks indicate the corresponding resonance conditions.

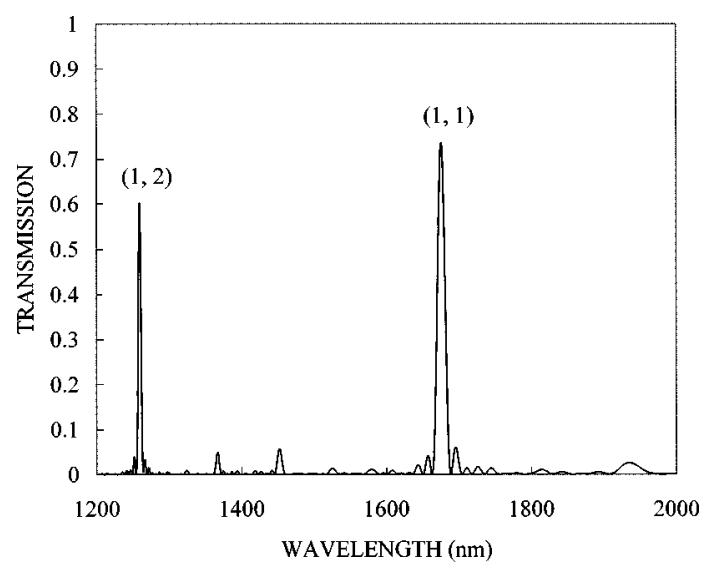

Fig. 15. Transmission spectrum of the $\nu=9$ cladding mode in a quasi-periodic Fibonacci LPG. The labels above the main peaks indicate the corresponding resonance conditions.

indicate the resonance peaks $\lambda_{\nu}^{(m, n)}$. The three main transmission peaks correspond to resonance conditions $(1,1),(0,1)$, and $(1,0)$. Figure 15 shows the transmission spectrum of the $\nu=9$ cladding mode. The two main peaks correspond to resonance conditions $(1,2)$ and $(1,1)$. By identifying each dip with the corresponding resonance conditions, we can see that the first group corresponds to the resonance condition with $(m, n)=(1,2)$, the second group corresponds to $(m, n)=(1,1)$, and the third group corresponds to $(m, n)=(0,1)$. There are also dips belonging not to these three groups but to other resonance conditions. By appropriately choosing the grating parameters, we can design a Fibonacci LPG for special applications. The detailed analysis and optimization of a Fibonacci LPG are under study.

\section{DISCUSSION}

Several approximations are made in the matrix method. The LPG is treated as a pure binary grating that is composed of distinct 1's and 0's. The transition region between regions 0 and 1 is reasonably assumed to be very short compared with the period of the grating. We also neglect the couplings to the backward-propagating modes, as discussed in the text. The couplings among longitudinal components and among different cladding modes are also neglected, as was pointed out by Erdogan in Ref. 7, since these approximations must be reconsidered for strong index modulation and couplings to fairly high-order cladding modes.

The transfer-matrix method provides an alternative approach to the theoretical study of LPG's and is especially useful in the case of quasi-periodic gratings such as the Fibonacci grating. The method can also be applied to the analysis and the design of specially patterned binary LPG's. Additionally, the matrix multiplication is efficient when implemented in computers. It should be noted that for apodized and continuously chirped gratings, the coupled-mode theory provides a better method than the transfer-matrix method. Which method is better should therefore depend on the physical situation and the application.

\section{CONCLUSIONS}

In summary, we have developed a transfer-matrix method based on the perturbation expansions. We use iterative substitutions to derive the vectorial perturbation expansions of the waveguide modes and then use the continuity condition of electric and magnetic fields to derive the transmission and reflection coefficients for incidence of modes on the interfaces of regions 0 and 1 . The results are then used to derive the $4 \times 4$ transfer matrix for two guided modes. By neglecting the reflection parts as discussed in the text, we can derive the forward transfer matrices to describe the codirectional couplings of waveguide modes.

The method is then applied to the study of the transmission spectrum of an ideal two-mode coupling grating, and analytic results are obtained by using Chebyshev's identities. We also derive the resonance condition that is equivalent to the phase-matching condition of coupledmode theory. Comparisons are made between a binary and a uniform sinusoidal grating coupler. Transmission spectra with different duty cycles are also studied.

By including the dispersion effect of a three-layered fiber waveguide, we apply the transfer-matrix method to calculate the transmission spectrum of a periodic binary LPG. The results are also compared with those of multimode coupled-mode theory, and excellent agreement is shown by considering the first-order Fourier expansion coefficient of the binary grating. The transfer matrices provide an efficient alternative method to calculate the spectra of LPG's.

The matrix method is applied to the study of a binary LPG with a quasi-periodic pattern. The general quasiperiodic LPG is defined based on the Bravis point set of a one-dimensional quasi-crystal. Each region 1 in the binary pattern is regarded as a diffractor. The resonance conditions are also derived by considering the interference of paths from all diffractors under the weak-coupling limit. The transmission spectrum of a Fibonacci LPG is calculated by using the matrix method. The Fibonacci lattice has been shown to have two incommensurate periods with ratio equal to the golden mean. Because of this property, the resonance conditions are parameterized by two integers and are denoted as $(m, n)$ in the text. The 
transmission dips are shown to be grouped, and each group corresponds to a specific resonance condition. Alternatively, each cladding mode contributes several dips in the transmission spectra, also as a result of the different resonance conditions.

\section{APPENDIX A: VECTORIAL PERTURBATION EXPANSION OF WAVEGUIDE MODES}

The main purpose of this appendix is to find the perturbation expansion to first order of the propagation constants and the mode fields. Our method is based on the following reciprocity theorem ${ }^{11}$ :

$$
\begin{aligned}
\beta_{\mathrm{j}}= & \beta_{\mathrm{j}}^{(0)} \\
& +\mathrm{k}_{0}\left(\frac{\epsilon_{0}}{\mu_{0}}\right)^{1 / 2} \frac{\mu \int_{\mathrm{A}_{\infty}}\left(\mathrm{n}^{2}-\overline{\mathrm{n}}^{2}\right) \mathbf{e}_{\mathrm{j}} \cdot \mathbf{e}_{\mathrm{j}}^{(0)^{*}} \mathrm{dA}}{\int_{\mathrm{A}_{\infty}}\left[\mathbf{e}_{\mathrm{j}} \times \mathbf{h}_{\mathrm{j}}^{(0)^{*}}+\mathbf{e}_{\mathrm{j}}^{(0)^{*}} \times \mathbf{h}_{\mathrm{j}}\right] \cdot \mathbf{z d A}} ;
\end{aligned}
$$

and the fields are expressed as

$$
\begin{aligned}
& \mathbf{e}_{\mathrm{tj}}=\sum_{\mathrm{k}}\left(\mathrm{a}_{\mathrm{jk}}+\mathrm{b}_{\mathrm{jk}}\right) \mathbf{e}_{\mathrm{tk}}^{(0)}, \\
& \mathbf{h}_{\mathrm{tj}}=\sum_{\mathrm{k}}\left(\mathrm{a}_{\mathrm{jk}}-\mathrm{b}_{\mathrm{jk}}\right) \mathbf{h}_{\mathrm{tk}}^{(0)}, \\
& \mathrm{e}_{\mathrm{zj}}=\frac{\overline{\mathrm{n}}^{2}}{\mathrm{n}^{2}} \sum_{\mathrm{k}}\left(\mathrm{a}_{\mathrm{jk}}-\mathrm{b}_{\mathrm{jk}}\right) \mathrm{e}_{\mathrm{zk}}^{(0)}, \\
& \mathrm{h}_{\mathrm{zj}}=\sum_{\mathrm{k}}\left(\mathrm{a}_{\mathrm{jk}}+\mathrm{b}_{\mathrm{jk}}\right) \mathrm{h}_{\mathrm{zk}}^{(0)},
\end{aligned}
$$

where the expansion coefficients are

$$
\begin{aligned}
\mathrm{a}_{\mathrm{jk}}= & \frac{\mathrm{k}_{0}}{4 \mathrm{~N}_{\mathrm{k}}^{(0)}\left[\beta_{\mathrm{j}}-\beta_{\mathrm{k}}^{(0)}\right]}\left(\frac{\epsilon_{0}}{\mu_{0}}\right)^{1 / 2} \mu \int_{\mathrm{A}_{\infty}}\left(\mathrm{n}^{2}-\overline{\mathrm{n}}^{2}\right) \mathbf{e}_{\mathrm{j}} \\
& \cdot \mathbf{e}_{\mathrm{k}}^{(0) *} \mathrm{dA}, \\
\mathrm{b}_{\mathrm{jk}}= & \frac{-\mathrm{k}_{0}}{4 \mathrm{~N}_{\mathrm{k}}^{(0)}\left[\beta_{\mathrm{j}}+\beta_{\mathrm{k}}^{(0)}\right]}\left(\frac{\epsilon_{0}}{\mu_{0}}\right)^{1 / 2} \mu \int_{\mathrm{A}_{\infty}}\left(\mathrm{n}^{2}-\overline{\mathrm{n}}^{2}\right) \mathbf{e}_{\mathrm{j}} \\
& \cdot \mathbf{e}_{-\mathrm{k}}^{(0) *} \mathrm{dA},
\end{aligned}
$$

where $\mathbf{e}_{-k}^{(0)} \equiv \mathbf{e}_{\mathrm{tk}}^{(0)}-\mathbf{z e}_{\mathrm{zk}}^{(0)}$. The above formulas [Eqs. (A1)-(A3)] are exact. The two problems in the above formulas are the unperturbed and perturbed mode fields and the corresponding propagation constants. Our main purpose is to find the first-order (i.e., the $\mu^{1}$-order) correction. Higher-order perturbations can be found by using the standard perturbation technique. We will describe the general method used to find the results but only list explicitly the first-order perturbation. I terative substitutions are used to derive the perturbative expansion. Let us expand the perturbed mode fields and propagation constants as follows:

$$
\begin{aligned}
& \beta_{\mathrm{j}}=\beta_{\mathrm{j}}^{(0)}+\mu \beta_{\mathrm{j}}^{(1)}+\mu^{2} \beta_{\mathrm{j}}^{(2)}+\cdots, \\
& \mathbf{e}_{\mathrm{j}}=\mathbf{e}_{\mathrm{j}}^{(0)}+\mu \mathbf{e}_{\mathrm{j}}^{(1)}+\mu^{2} \mathbf{e}_{\mathrm{j}}^{(2)}+\cdots, \\
& \mathbf{h}_{\mathrm{j}}=\mathbf{h}_{\mathrm{j}}^{(0)}+\mu \mathbf{h}_{\mathrm{j}}^{(1)}+\mu^{2} \mathbf{h}_{\mathrm{j}}^{(2)}+\cdots .
\end{aligned}
$$

To find the shift of the propagation constant, we substitute E qs. (A4) into Eq. (A1) for the fields and collect terms of the same order; we then have the first-order correction:

$$
\beta_{\mathrm{j}}^{(1)}=\frac{\mathrm{k}_{0}}{4 \mathrm{~N}_{\mathrm{j}}^{(0)}}\left(\frac{\epsilon_{0}}{\mu_{0}}\right)^{1 / 2} \int_{\mathrm{A}_{\infty}}\left(\mathrm{n}^{2}-\overline{\mathrm{n}}^{2}\right) \mathbf{e}_{\mathrm{j}}^{(0)} \cdot \mathbf{e}_{\mathrm{j}}^{(0)^{*}} \mathrm{dA} .
$$

To find the first-order perturbation of the mode fields, we substitute Eqs. (A4) into Eqs. (A3). It can be found that, for $j \neq k$,

$$
\begin{aligned}
a_{j k}= & \mu a_{j k}^{(1)}+\mu^{2} a_{j k}^{(2)}+\cdots, \quad a_{j k}^{(0)}=0, \\
a_{j k}^{(1)}= & \frac{k_{0}}{4 N_{k}^{(0)}\left[\beta_{j}^{(0)}-\beta_{k}^{(0)}\right]}\left(\frac{\epsilon_{0}}{\mu_{0}}\right)^{1 / 2} \\
& \times \int_{A_{\infty}}\left(n^{2}-\bar{n}^{2}\right) \mathbf{e}_{j}^{(0)} \cdot \mathbf{e}_{k}^{(0)^{*}} d A, \\
b_{j k}= & \mu b_{j k}^{(1)}+\mu^{2} b_{j k}^{(2)}+\cdots, \quad b_{j k}^{(0)}=0, \\
b_{j k}^{(1)}= & \frac{-k_{0}}{4 N_{k}^{(0)}\left[\beta_{j}^{(0)}+\beta_{k}^{(0)}\right]}\left(\frac{\epsilon_{0}}{\mu_{0}}\right)^{1 / 2} \\
& \times \int_{A_{\infty}}\left(n^{2}-\bar{n}^{2}\right) \mathbf{e}_{j}^{(0)} \cdot \mathbf{e}_{-k}^{(0) *} d A .
\end{aligned}
$$

We also have for $b_{j j}=\mu b_{j j}^{(1)}+\mu^{2} b_{j j}^{(2)}+\cdots$ the following first-order correction:

$$
\begin{aligned}
& \mathrm{b}_{\mathrm{j} j}^{(0)}=0, \\
& \mathrm{~b}_{\mathrm{j} j}^{(1)}=\frac{-\mathrm{k}_{0}}{4 \mathrm{~N}_{\mathrm{j}}^{(0)} 2 \beta_{\mathrm{j}}^{(0)}}\left(\frac{\epsilon_{0}}{\mu_{0}}\right)^{1 / 2} \int_{\mathrm{A}_{\infty}}\left(\mathrm{n}^{2}-\overline{\mathrm{n}}^{2}\right) \mathbf{e}_{\mathrm{j}}^{(0)} \cdot \mathbf{e}_{-\mathrm{j}}^{(0) *} \mathrm{dA} .
\end{aligned}
$$

Using the formula for $\beta_{j}^{(1)}$, we can prove that

$$
a_{j j}^{(0)}=1 \text {. }
$$

Now we use the above first-order corrections to find the new normalization constant:

$$
\begin{aligned}
\mathbf{N}_{\mathrm{j}}^{(1)}= & \frac{1}{2} \int_{\mathrm{A}_{\infty}}\left\{\left[\mathbf{e}_{\mathrm{tj}}^{(0)}+\mu \mathbf{e}_{\mathrm{tj}}^{(1)}+\mu^{2} \mathbf{e}_{\mathrm{t} j}^{(2)}\right]\right. \\
& \left.\times\left[\mathbf{h}_{\mathrm{tj}}^{(0)}+\mu \mathbf{h}_{\mathrm{tj}}^{(1)}+\mu^{2} \mathbf{h}_{\mathrm{tj}}^{(2)}\right]\right\} \cdot \mathbf{z} \mathrm{dA} \\
= & {\left[1+2 \mu \mathrm{a}_{\mathrm{jj}}^{(1)}+2 \mu^{2} \mathrm{a}_{\mathrm{j} j}^{(2)}\right] \mathbf{N}_{\mathrm{j}}^{(0)} } \\
& +\mu^{2} \sum_{\mathrm{k}}\left[\left|\mathrm{a}_{\mathrm{jk}}^{(1)}\right|^{2}-\left|\mathrm{b}_{\mathrm{jk}}^{(1)}\right|^{2}\right] \mathrm{N}_{\mathrm{k}}^{(0)} .
\end{aligned}
$$

Here we consider terms up to the second-order perturbation. If we require the mode to be normal ized to unity for all orders [so $\mathrm{N}_{\mathrm{j}}^{(0)}=1$ for all $\mathrm{j}$ ], the mode field must be renormalized as follows. Let $Z_{j}$ be defined as

$$
Z_{j}=1+2 \mu a_{j j}^{(1)}+2 \mu^{2} a_{j j}^{(2)}+\mu^{2} \sum_{k}\left[\left|a_{j k}^{(1)}\right|^{2}-\left|b_{j k}^{(1)}\right|^{2}\right] .
$$


The fields are normalized according to $\mathbf{e}_{\mathrm{i}} \rightarrow \mathbf{e}_{\mathrm{j}} / \sqrt{Z_{\mathrm{j}}}$ and $\mathbf{h}_{\mathrm{j}} \rightarrow \mathbf{h}_{\mathrm{j}} / \sqrt{Z_{\mathrm{j}}}$. Thus, to lowest order, we have to replace Eq. (A9) by the following:

$$
\begin{aligned}
a_{j j}^{(0)}= & 1 / \sqrt{Z_{j}} \cong 1-\frac{1}{2}\left\{2 \mu a_{j j}^{(1)}+2 \mu^{2} a_{j j}^{(2)}\right. \\
& \left.+\mu^{2} \sum_{k}\left[\left|a_{j k}^{(1)}\right|^{2}-\left|b_{j k}^{(1)}\right|^{2}\right]\right\} .
\end{aligned}
$$

To satisfy the power conservation condition to second order, we should have

$$
1=\left|a_{j j}^{(0)}\right|^{2}+\mu^{2}\left\{\left|b_{j j}^{(1)}\right|^{2}+\sum_{k \neq j}\left[\left|a_{j k}^{(1)}\right|^{2}+\left|b_{j k}^{(1)}\right|^{2}\right]\right\} .
$$

Thus we obtain the following result for first-order and second-order corrections to $\mathrm{a}_{\mathrm{jj}}$ :

$$
a_{j j}^{(1)}=0, \quad a_{j j}^{(2)}=\sum_{k}\left|b_{j k}^{(1)}\right|^{2} .
$$

(A16)

The self-transmission coefficient to second order can be written as

$$
\begin{aligned}
a_{j j} & \equiv 1-\frac{1}{2} \mu^{2} D_{j} \\
& =1-\frac{1}{2} \mu^{2}\left\{\left|b_{j j}^{(1)}\right|^{2}+\sum_{k \neq j}\left[\left|a_{j k}^{(1)}\right|^{2}+\left|b_{j k}^{(1)}\right|^{2}\right]\right\} .
\end{aligned}
$$

Here we defined a quantity $D_{j} / 2$, which represents the power loss from the original mode.

\section{APPENDIX B: TRANSFER-MATRIX ELEMENTS}

In this appendix the elements of the $4 \times 4$ transfer matrix will be derived to second order in perturbation expansions. Consider the process for incidence of mode $\mathrm{k}$ from region 0 to region 1 shown in Fig. 2; the perturbation solutions (15) result in the following relations among mode amplitudes:

$$
\left(\begin{array}{c}
1 \\
\mu \mathrm{b}_{\mathrm{kk}}^{(1)} \\
0 \\
\mu \mathrm{b}_{\mathrm{kj}}^{(1)}
\end{array}\right)=[\mathrm{F}]\left(\begin{array}{c}
1-\frac{\mu^{2}}{2} \mathrm{D}_{\mathrm{k}} \\
0 \\
-\mu \mathrm{a}_{\mathrm{kj}}^{(1)} \\
0
\end{array}\right)
$$

Similarly, for the process of incidence of mode $\mathrm{j}$ from region 0 to region 1 , we have

$$
\left(\begin{array}{c}
0 \\
\mu \mathrm{b}_{\mathrm{jk}}^{(1)} \\
1 \\
\mu \mathrm{b}_{\mathrm{jj}}^{(1)}
\end{array}\right)=[\mathrm{F}]\left(\begin{array}{c}
-\mu \mathrm{a}_{\mathrm{jk}}^{(1)} \\
0 \\
1-\frac{\mu^{2}}{2} \mathrm{D}_{\mathrm{j}} \\
0
\end{array}\right) .
$$

We pick the first rows in Eqs. (B1) and (B2); then we obtain

$$
\begin{aligned}
& 1=F_{11}\left(1-\frac{\mu^{2}}{2} D_{k}\right)+F_{13}\left[-\mu a_{k j}^{(1)}\right], \\
& 0=F_{11}\left[-\mu a_{j k}^{(1)}\right]+F_{13}\left(1-\frac{\mu^{2}}{2} D_{j}\right) .
\end{aligned}
$$

This set of equations is then sufficient to solve the matrix elements $F_{11}$ and $F_{13}$. The others are derived similarly. The 16 matrix elements to second order in $\mu$ are listed in the following:

$$
\begin{aligned}
& \mathrm{F}_{11}=1+\mu^{2}\left\{\frac{\kappa_{\mathrm{kj}} \kappa_{\mathrm{jk}}}{\left[\beta_{\mathrm{j}}^{(0)}-\beta_{\mathrm{k}}^{(0)}\right]^{2}}+\frac{1}{2} \mathrm{D}_{\mathrm{k}}\right\}, \\
& \mathrm{F}_{12}=\mu \frac{-\kappa_{\mathrm{kk}}^{\prime}}{2 \beta_{\mathrm{k}}^{(0)}}+\mu^{2} \frac{-\kappa_{\mathrm{kj}} \kappa_{\mathrm{jk}}^{\prime}}{\left[\beta_{\mathrm{j}}^{(0)}-\beta_{\mathrm{k}}^{(0)}\right]\left[\beta_{\mathrm{j}}^{(0)}+\beta_{\mathrm{k}}^{(0)}\right]}, \\
& \mathrm{F}_{13}=\mu \frac{\kappa_{\mathrm{kj}}}{\beta_{\mathrm{j}}^{(0)}-\beta_{\mathrm{k}}^{(0)}},
\end{aligned}
$$$$
\mathrm{F}_{14}=\mu \frac{-\kappa_{\mathrm{kj}}^{\prime}}{\beta_{\mathrm{j}}^{(0)}+\beta_{\mathrm{k}}^{(0)}}+\mu^{2} \frac{-\kappa_{\mathrm{kj}} \kappa_{\mathrm{jj}}^{\prime}}{2 \beta_{\mathrm{j}}^{(0)}\left[\beta_{\mathrm{j}}^{(0)}-\beta_{\mathrm{k}}^{(0)}\right]},
$$$$
\mathrm{F}_{21}=\mu \frac{-\kappa_{\mathrm{kk}}^{\prime}}{2 \beta_{\mathrm{k}}^{(0)}}+\mu^{2} \frac{-\kappa_{\mathrm{kj}}^{\prime} \kappa_{\mathrm{jk}}}{\left[\beta_{\mathrm{j}}^{(0)}-\beta_{\mathrm{k}}^{(0)}\left[\beta_{\mathrm{j}}^{(0)}+\beta_{\mathrm{k}}^{(0)}\right]\right.},
$$$$
\mathrm{F}_{22}=1+\mu^{2}\left\{\frac{\kappa_{\mathrm{kk}}^{\prime}{ }^{2}}{\left[2 \beta_{\mathrm{k}}^{(0)}\right]^{2}}+\frac{\kappa_{\mathrm{kj}}^{\prime} \kappa_{\mathrm{j} k}^{\prime}}{\left[\beta_{\mathrm{j}}^{(0)}+\beta_{\mathrm{k}}^{(0)}\right]^{2}}-\frac{1}{2} \mathrm{D}_{\mathrm{k}}\right\},
$$

$$
\begin{aligned}
& \mathrm{F}_{23}= \mu \frac{-\kappa_{\mathrm{kj}}^{\prime}}{\beta_{\mathrm{j}}^{(0)}+\beta_{\mathrm{k}}^{(0)}}+\mu^{2} \frac{-\kappa_{\mathrm{kk}}^{\prime} \kappa_{\mathrm{kj}}}{2 \beta_{\mathrm{k}}^{(0)}\left[\beta_{\mathrm{j}}^{(0)}-\beta_{\mathrm{k}}^{(0)}\right]}, \\
& \mathrm{F}_{24}=\mu \frac{\kappa_{\mathrm{kj}}}{\beta_{\mathrm{j}}^{(0)}-\beta_{\mathrm{k}}^{(0)}}+\mu^{2}\left\{\frac{\kappa_{\mathrm{kk}}^{\prime} \kappa_{\mathrm{kj}}^{\prime}}{2 \beta_{\mathrm{k}}^{(0)}\left[\beta_{\mathrm{j}}^{(0)}+\beta_{\mathrm{k}}^{(0)}\right]}\right. \\
&\left.+\frac{\kappa_{\mathrm{kj}}^{\prime} \kappa_{\mathrm{jj}}^{\prime}}{2 \beta_{\mathrm{j}}^{(0)}\left[\beta_{\mathrm{j}}^{(0)}+\beta_{\mathrm{k}}^{(0)}\right]}\right\},
\end{aligned}
$$

$$
\begin{aligned}
& \mathrm{F}_{31}=\mu \frac{\kappa_{\mathrm{jk}}}{\beta_{\mathrm{k}}^{(0)}-\beta_{\mathrm{j}}^{(0)}}, \\
& \mathrm{F}_{32}=\mu \frac{-\kappa_{\mathrm{jk}}^{\prime}}{\beta_{\mathrm{k}}^{(0)}+\beta_{\mathrm{j}}^{(0)}}+\mu^{2} \frac{-\kappa_{\mathrm{jk}} \kappa_{\mathrm{kk}}^{\prime}}{2 \beta_{\mathrm{k}}^{(0)}\left[\beta_{\mathrm{k}}^{(0)}-\beta_{\mathrm{j}}^{(0)}\right]},
\end{aligned}
$$

$\mathrm{F}_{33}=1+\mu^{2}\left\{\frac{\kappa_{\mathrm{jk}} \kappa_{\mathrm{kj}}}{\left[\beta_{\mathrm{k}}^{(0)}-\beta_{\mathrm{j}}^{(0)}\right]^{2}}+\frac{1}{2} \mathrm{D}_{\mathrm{j}}\right\}$,

$\mathrm{F}_{34}=\mu \frac{-\kappa_{\mathrm{jj}}^{\prime}}{2 \beta_{\mathrm{j}}^{(0)}}+\mu^{2} \frac{-\kappa_{\mathrm{jk}} \kappa_{\mathrm{kj}}^{\prime}}{\left[\beta_{\mathrm{k}}^{(0)}-\beta_{\mathrm{j}}^{(0)}\right]\left[\beta_{\mathrm{k}}^{(0)}+\beta_{\mathrm{j}}^{(0)}\right]}$,

$$
\mathrm{F}_{41}=\mu \frac{-\kappa_{\mathrm{jk}}^{\prime}}{\beta_{\mathrm{k}}^{(0)}+\beta_{\mathrm{j}}^{(0)}}+\mu^{2} \frac{-\kappa_{\mathrm{jj}}^{\prime} \kappa_{\mathrm{jk}}}{2 \beta_{\mathrm{j}}^{(0)}\left[\beta_{\mathrm{k}}^{(0)}+\beta_{\mathrm{j}}^{(0)}\right]},
$$




$$
\begin{aligned}
\mathrm{F}_{42}= & \mu \frac{\kappa_{\mathrm{jk}}}{\beta_{\mathrm{k}}^{(0)}-\beta_{\mathrm{j}}^{(0)}}+\mu^{2}\left\{\frac{\kappa_{\mathrm{jk}}^{\prime} \kappa_{\mathrm{kk}}^{\prime}}{2 \beta_{\mathrm{k}}^{(0)}\left[\beta_{\mathrm{k}}^{(0)}+\beta_{\mathrm{j}}^{(0)}\right]}\right. \\
& \left.+\frac{\kappa_{\mathrm{jj}}^{\prime} \kappa_{\mathrm{jk}}^{\prime}}{2 \beta_{\mathrm{j}}^{(0)}\left[\beta_{\mathrm{k}}^{(0)}+\beta_{\mathrm{j}}^{(0)}\right]}\right\}, \\
\mathrm{F}_{43}= & \mu \frac{-\kappa_{\mathrm{jj}}^{\prime}}{2 \beta_{\mathrm{j}}^{(0)}}+\mu^{2} \frac{\left[\beta_{\mathrm{k}}^{(0)}-\beta_{\mathrm{j}}^{(0)}\right]\left[\beta_{\mathrm{k}}^{(0)}+\beta_{\mathrm{j}}^{(0)}\right]}{\left[{ }^{(0)}\right.} \\
\mathrm{F}_{44}= & 1+\mu^{2}\left\{\frac{\kappa_{\mathrm{jj}}^{2}}{\left[2 \beta_{\mathrm{j}}^{(0)}\right]^{2}}+\frac{\kappa_{\mathrm{jk}}^{\prime} \kappa_{\mathrm{kj}}^{\prime}}{\left[\beta_{\mathrm{k}}^{(0)}+\beta_{\mathrm{j}}^{(0)}\right]^{2}}-\frac{1}{2} \mathrm{D}_{\mathrm{j}}\right\} .
\end{aligned}
$$

\section{APPENDIX C: HIGHLIGHT OF COUPLED-MODE THEORY}

The derivation and the solution of the coupled-mode theory can be found in many standard textbooks. ${ }^{5,11}$ Here we will highlight the theory and list the solution for ideal two-mode coupling. To be consistent with our notation, the coupled-mode equation for the amplitude of mode $\mathrm{k}$ is

$$
\frac{d A_{k}}{d z}=i \beta_{k}^{(1)} A_{k}+\sum_{j} \mathrm{i} \kappa_{k j} A_{j} \exp \left(-i 2 \delta_{k j} z\right),
$$

where $\beta_{\mathrm{k}}^{(1)}$ is the first-order correction to the propagation constant, which is due to the dc term in the photoinduced index modulation, and $\kappa_{\mathrm{kj}}$ is the coupling coefficient of mode $k$ and mode $j$, which is due to the ac part of the index modulation. The detuning parameter $\delta_{\mathrm{kj}}$ is defined as

$$
\delta_{\mathrm{kj}}=-\delta_{\mathrm{jk}} \equiv \frac{1}{2}\left[\beta_{\mathrm{k}}^{(0)}-\beta_{\mathrm{j}}^{(0)}-\frac{2 \pi}{\Lambda}\right] .
$$

Other quantities are defined in the text. For a uniform sinusoidal LPG with two-mode coupling (e.g., let mode k be the core mode LP $P_{01}$, and let mode $j$ be one specific cladding mode $\mathrm{HE}_{1 \nu}$ ), there is an analytic solution for $\mathrm{Eq}$. (C1), which can be expressed in the matrix form

$$
\begin{aligned}
& \left(\begin{array}{l}
A_{k}\left(L_{g}\right) \\
A_{j}\left(L_{g}\right)
\end{array}\right) \\
& =\left[\begin{array}{cc}
\cos \left(k L_{g}\right)+i \frac{\delta}{k} \sin \left(k L_{g}\right) & \operatorname{i} \frac{\kappa}{k} \sin \left(k L_{g}\right) \\
i \frac{\kappa^{*}}{k} \sin \left(k L_{g}\right) & \cos \left(k L_{g}\right)+i \frac{\delta}{k} \sin \left(k L_{g}\right)
\end{array}\right] \\
& \quad \times\left(\begin{array}{c}
A_{k}(0) \\
A_{j}(0)
\end{array}\right),
\end{aligned}
$$

where $\delta \equiv \delta_{\mathrm{kj}}+\left[\beta_{\mathrm{k}}^{(1)}-\beta_{\mathrm{j}}^{(1)}\right] / 2$ is the modified detuning parameter, $\kappa \equiv \kappa_{\mathrm{kj}}$ is the coupling coefficient, and $\mathrm{k}$ $\equiv \sqrt{\delta^{2}+|\kappa|^{2}}$. The coupled-mode theory has been successfully applied to describe the cladding-mode resonance of a sinusoidal modulated LPG in Ref. 7. Under certain approximations, also discussed in the same reference, the coupled-mode equations that describe copropagating couplings in a LPG take the following form:

$$
\begin{aligned}
\frac{\mathrm{dA}^{\mathrm{co}}}{\mathrm{dz}}= & \mathrm{i} \kappa_{01-01}^{\mathrm{co}-\mathrm{co}} \mathrm{A}^{\mathrm{co}} \\
& +\mathrm{i} \sum_{\nu} \frac{\mathrm{m}}{2} \kappa_{1 \nu-01}^{\mathrm{cl}-\mathrm{co}} \mathrm{A}_{\nu}^{\mathrm{cl}} \exp \left(-\mathrm{i} 2 \delta_{1 \nu-01}^{\mathrm{cl}-\mathrm{co} z)}\right. \\
\sum_{\nu}\left[\frac{\mathrm{dA}{ }_{\nu}^{\mathrm{cl}}}{\mathrm{dz}}=\right. & \left.+\mathrm{i} \frac{\mathrm{m}}{2} \kappa_{1 \nu-01}^{\mathrm{dl}-\mathrm{co}} \mathrm{A}^{\mathrm{co}} \exp \left(+\mathrm{i} 2 \delta_{1 \nu-01}^{\mathrm{cl}-\mathrm{co}} \mathrm{z}\right)\right]
\end{aligned}
$$

where $\mathrm{m}$ is the depth of the index modulation. The summation sign in Eq. (C5) signifies that the equation is applicable to all cladding modes with varying mode number $\nu$. Note that in the above equations the photoinduced index change used in the calculation of the various coupling coefficients is the averaged dc value.

Address correspondence to L on A. Wang at the location on the title page or by phone, 886-223-635251; fax, 886223-656327; or e-mail, Ion@ccms.ntu.edu.tw.

\section{REFERENCES}

1. G. Meltz, W. W. Morey, and W. H. Glenn, "Formation of Bragg gratings in optical fibers by a transverse holographic method," Opt. Lett. 14, 823-825 (1989).

2. A. M. Vengsarkar, P. J . Lemaire, J . B. J udkins, V. Bhatia, T. Erdogan, and J. E. Sipe, "Long-period fiber gratings as band-rejection filters," J. Lightwave Technol. 14, 58-65 (1996).

3. A. M. Vengsarkar, J . R. Pedrazzani, J . B. J udkins, P. J . Lemaire, N. S. Bergano, and C. R. Davidson, "Long-period fiber-grating-based gain equalizers," Opt. Lett. 21, 336-338 (1996).

4. V. Bhatia and A. M. Vengsarkar, "Optical fiber long-period grating sensors," Opt. Lett. 21, 692-694 (1996).

5. D. Marcuse, Theory of Dielectric Optical Waveguides (Academic, Boston, 1991).

6. V. Mizrahi and J . E. Sipe, "Optical properties of photosensitive fiber phase gratings," J. Lightwave Technol. 11, 1513-1517 (1993).

7. T. Erdogan, "Cladding-mode resonances in short- and longperiod fiber grating filters," J. Opt. Soc. Am. A 14, 17601773 (1997).

8. P. Yeh, Optical Waves in Layered Media (Wiley, New York, 1988).

9. H. A. Macleod, Thin-Film Optical Filters (American Elsevier, New York, 1969).

10. P. Yeh, "Electromagnetic propagation in birefringent layered media," J. Opt. Soc. Am. 69, 742-756 (1979).

11. A. W. Snyder and J. D. Love, Optical Waveguide Theory (Chapman \& Hall, London, 1991), Secs. 31-1 and 31-8.

12. A. W. Snyder, "Excitation and scattering of modes on a dielectric or optical fiber," IEEE Trans. Microwave Theory Tech. MTT-17, 1138-1144 (1969).

13. D. Levine and P. J. Steinhardt, "Quasicrystals: a new class of ordered structures," Phys. Rev. Lett. 53, 2477-2480 (1984).

14. R. Merlin, K. Bajema, R. Clarke, F.-Y. J uang, and P. K. Bhattacharya, "Quasiperiodic GaAs-AIAs heterostructures," Phys. Rev. Lett. 55, 1768-1771 (1985).

15. D. Levine and P. J . Steinhardt, "Quasicrystals. I. Definitions and structure," Phys. Rev. B 34, 596-616 (1986). 